\title{
A LAGRANGIAN APPROACH FOR THE COMPRESSIBLE NAVIER-STOKES EQUATIONS
}

\author{
UNE APPROCHE LAGRANGIENNE POUR LE SYSTÈME DE NAVIER-STOKES \\ COMPRESSIBLE
}

\author{
RAPHAËL DANCHIN
}

\begin{abstract}
Here we investigate the Cauchy problem for the barotropic Navier-Stokes equations in $\mathbb{R}^{n}$, in the critical Besov spaces setting. We improve recent results (see $[4,8,9]$ ) as regards the uniqueness condition: initial velocities in critical Besov spaces with (not too) negative indices generate a unique local solution. Apart from (critical) regularity, the initial density just has to be bounded away from 0 and to tend to some positive constant at infinity. Densitydependent viscosity coefficients may be considered. Using Lagrangian coordinates is the key to our statements as it enables us to solve the system by means of the basic contraction mapping theorem. As a consequence, conditions for uniqueness are the same as for existence, and Lipschitz continuity of the flow map (in Lagrangian coordinates) is established.

RÉSUMÉ. On étudie le problème de Cauchy pour le système de Navier-Stokes barotrope dans $\mathbb{R}^{n}$, avec régularité Besov critique. On affaiblit la condition d'unicité des articles $[4,8,9]$, ce qui permet d'établir entre autres que des vitesses initiales ayant une régularité Besov (pas trop) négative génèrent une solution unique. La densité initiale est à régularité critique et doit juste être strictement positive et tendre vers une constante à l'infini. Les coefficients de viscosité preuvent dépendre de la densité. L'usage de coordonnées lagrangiennes est la clef de toutes ces améliorations car il permet de résoudre le système par itérations de Picard. Comme corollaire immédiat, on obtient que les conditions pour l'unicité sont les mêmes que pour l'existence, ainsi que la continuité de l'opérateur solution (pour le système écrit en coordonnées lagrangiennes).
\end{abstract}

\section{INTRODUCTION}

We address the well-posedness issue for the barotropic compressible Navier-Stokes equations with variable density in the whole space $\mathbb{R}^{n}$ :

$$
\left\{\begin{array}{l}
\partial_{t} \rho+\operatorname{div}(\rho u)=0, \\
\partial_{t}(\rho u)+\operatorname{div}(\rho u \otimes u)-2 \operatorname{div}(\mu(\rho) D(u))-\nabla(\lambda(\rho) \operatorname{div} u)+\nabla(P(\rho))=0, \\
\left.\rho\right|_{\mid t=0}=\rho_{0},\left.\quad u\right|_{\mid t=0}=u_{0} .
\end{array}\right.
$$

Above $\rho=\rho(t, x) \in \mathbb{R}_{+}$stands for the density, $u=u(t, x) \in \mathbb{R}^{n}$, for the velocity field. The space variable $x$ belongs to the whole $\mathbb{R}^{n}$. The notation $D(u)$ designates the deformation tensor which is defined by

$$
D(u):=\frac{1}{2}(D u+\nabla u) \quad \text { with } \quad(D u)_{i j}:=\partial_{j} u^{i} \quad \text { and } \quad(\nabla u)_{i j}:=\partial_{i} u^{j} .
$$

The pressure function $P$ and the viscosity coefficients $\lambda$ and $\mu$ are given suitably smooth functions of the density. With no loss of generality, one may assume that $P$ is defined over $\mathbb{R}$

Date: October 24, 2018.

1991 Mathematics Subject Classification. 35Q35, 76N10.

Key words and phrases. Compressible fluids, uniqueness, critical regularity, Lagrangian coordinates.

Fluides compressibles, unicité, régularité critique, coordonnées lagrangiennes. 
and vanishes at 0 . As we focus on viscous fluids, we suppose that

$$
\alpha:=\min \left(\inf _{\rho>0}(\lambda(\rho)+2 \mu(\rho)), \inf _{\rho>0} \mu(\rho)\right)>0,
$$

which ensures the second order operator in the velocity equation of (0.1) to be uniformly elliptic.

We supplement System (0.1) with the condition at infinity that $u$ tends to 0 and $\rho$, to some positive constant (that may be taken equal to 1 after suitable normalization). The exact meaning of those boundary conditions will be given by the functional framework in which we shall consider the system.

In the present paper, we aim at solving (0.1) in critical functional spaces, that is in spaces which have the same invariance with respect to time and space dilation as the system itself (see e.g. [8] for more explanations about this nowadays classical approach). In this framework, it has been stated $[8,9]$ in the constant coefficients case that, for data $\left(\rho_{0}, u_{0}\right)$ such that

$$
a_{0}:=\left(\rho_{0}-1\right) \in \dot{B}_{p, 1}^{n / p}\left(\mathbb{R}^{n}\right), \quad u_{0} \in \dot{B}_{p, 1}^{n / p-1}\left(\mathbb{R}^{n}\right)
$$

and that, for a small enough constant $c$,

$$
\left\|a_{0}\right\|_{\dot{B}_{p, 1}^{n / p}\left(\mathbb{R}^{n}\right)} \leq c
$$

we have for any $p \in[1,2 n)$ :

- existence of a local solution $(\rho, u)$ such that $a:=(\rho-1) \in \mathcal{C}_{b}\left([0, T] ; \dot{B}_{p, 1}^{n / p}\right), \quad u \in$ $\mathcal{C}_{b}\left([0, T] ; \dot{B}_{p, 1}^{n / p-1}\right)$ and $\partial_{t} u, \nabla^{2} u \in L^{1}\left(0, T ; \dot{B}_{p, 1}^{n / p-1}\right)$;

- uniqueness in the above space if in addition $p \leq n$.

If $p \leq n$ then the viscosity coefficients may depend (smoothly) on $\rho$ and the smallness condition (0.3) may be replaced by the following positivity condition (see $[4,10]$ ):

$$
\inf _{x \in \mathbb{R}^{n}} \rho_{0}(x)>0 .
$$

Those results have been somewhat extended in [17] where it has been noticed that $a_{0}$ may be taken in a larger Besov space, with another Lebesgue exponent.

The above results are based on maximal regularity estimates in Besov spaces for the evolutionary Lamé system, and on the Schauder-Tychonoff fixed point theorem. In effect, owing to the hyperbolicity of the density equation, there is a loss of one derivative in the stability estimates thus precluding the use of the contraction mapping (or Banach fixed point) theorem. As a consequence, with this method it is found that the conditions for uniqueness are stronger than those for existence.

Following our recent paper [14] dedicated to the incompressible density-dependent NavierStokes equation, and older works concerning the compressible Navier-Stokes equations (see [21, $22,23]$ ), we here aim at solving System (0.1) in the Lagrangian coordinates. The main motivation is that the mass is constant along the flow hence, to some extent, only the (parabolic type) equation for the velocity has to be considered. After performing this change of coordinates, we shall see that solving (0.1) may be done by means of the Banach fixed point theorem. Hence, the condition for uniqueness is the same as that for the existence, and the flow map is Lipschitz continuous. In addition, in the case of fully nonhomogeneous fluids with variable viscosity coefficients, the analysis turns out to be simpler than in $[4,10]$ even for density-dependent viscosity coefficients and in the case where the density is not close to a constant. Indeed, our proof relies essentially on a priori estimates for a parabolic system (a suitable linearization of the momentum equation in Lagrangian coordinates) with rough constant depending only on the initial density hence time-independent. In contrast, in $[4,10]$ tracking the time-dependency of the coefficients was quite technical. 
We now come to the plan of the paper. In the next section, we introduce the compressible Navier-Stokes equations in Lagrangian coordinates and present our main results. Section 2 is devoted to the proof of our main existence and uniqueness result in the simpler case where the density is close to a constant and the coefficients, density independent. In Section 3, we treat the general fully nonhomogeneous case with nonconstant coefficients. A great deal of the analysis is contained in the study of the linearized momentum equation for (0.1) (see Subsection 3.1) which turns out to be a Lamé type system with variable rough coefficients. This will enable us to define a self-map $\Phi$ on a suitably small ball of some Banach space $E_{p}(T)$ and to apply the contraction mapping theorem so as to solve the compressible Navier-Stokes equations in Lagrangian coordinates. In the Appendix we prove several technical results concerning the Lagrangian coordinates and Besov spaces.

Notation: Throughout, the notation $C$ stands for a generic constant (the meaning of which depends on the context), and we sometimes write $A \lesssim B$ instead of $A \leq C B$. For $X$ a Banach space, $p \in[1,+\infty]$ and $T>0$, the notation $L^{p}(0, T ; X)$ or $L_{T}^{p}(X)$ designates the set of measurable functions $f:[0, T] \rightarrow X$ with $t \mapsto\|f(t)\|_{X}$ in $L^{p}(0, T)$, endowed with the norm

$$
\|f\|_{L_{T}^{p}(X)}:=\|\| f\left\|_{X}\right\|_{L^{p}(0, T)} .
$$

We agree that $\mathcal{C}([0, T] ; X)$ denotes the set of continuous functions from $[0, T]$ to $X$.

\section{Main Results}

Before deriving the Lagrangian equations corresponding to (0.1), let us introduce more notation. We agree that for a $C^{1}$ function $F: \mathbb{R}^{n} \rightarrow \mathbb{R}^{n} \times \mathbb{R}^{m}$ then $\operatorname{div} F: \mathbb{R}^{n} \rightarrow \mathbb{R}^{m}$ with

$$
(\operatorname{div} F)^{j}:=\sum_{i} \partial_{i} F_{i j} \quad \text { for } 1 \leq j \leq m,
$$

and that for $A=\left(A_{i j}\right)_{1 \leq i, j \leq n}$ and $B=\left(B_{i j}\right)_{1 \leq i, j \leq n}$ two $n \times n$ matrices, we denote

$$
A: B=\operatorname{Tr} A B=\sum_{i, j} A_{i j} B_{j i}
$$

The notation $\operatorname{adj}(A)$ designates the adjugate matrix that is the transposed cofactor matrix. Of course if $A$ is invertible then we have $\operatorname{adj}(A)=(\operatorname{det} A) A^{-1}$. Finally, given some matrix $A$, we define the "twisted" deformation tensor and divergence operator (acting on vector fields $z$ ) by the formulae

$$
D_{A}(z):=\frac{1}{2}\left(D z \cdot A+{ }^{T} A \cdot \nabla z\right) \text { and } \operatorname{div}_{A} z:={ }^{T} A: \nabla z=D z: A .
$$

Let $X$ be the flow associated to the vector-field $u$, that is the solution to

$$
X(t, y)=y+\int_{0}^{t} u(\tau, X(\tau, y)) d \tau .
$$

Denoting

$$
\bar{\rho}(t, y):=\rho(t, X(t, y)) \text { and } \bar{u}(t, y)=u(t, X(t, y))
$$

with $(\rho, u)$ a solution of $(0.1)$, and using the chain rule and Lemma 1 from the Appendix, we gather that $(\bar{\rho}, \bar{u})$ satisfies

$$
\left\{\begin{array}{l}
\partial_{t}(J \bar{\rho})=0 \\
\rho_{0} \partial_{t} \bar{u}-\operatorname{div}\left(\operatorname{adj}(D X)\left(2 \mu(\bar{\rho}) D_{A}(\bar{u})+\lambda(\bar{\rho}) \operatorname{div}_{A} \bar{u} \operatorname{Id}+P(\bar{\rho}) \operatorname{Id}\right)\right)=0
\end{array}\right.
$$


with $J:=\operatorname{det} D X$ and $A:=\left(D_{y} X\right)^{-1}$. Note that one may forget any reference to the initial Eulerian vector-field $u$ by defining directly the "flow" $X$ of $\bar{u}$ by the formula

$$
X(t, y)=y+\int_{0}^{t} \bar{u}(\tau, y) d \tau .
$$

We want to solve the above system in critical homogeneous Besov spaces. Let us recall that, for $1 \leq p \leq \infty$ and $s \leq n / p$, a tempered distribution $u$ over $\mathbb{R}^{n}$ belongs to the homogeneous Besov space $\dot{B}_{p, 1}^{s}\left(\mathbb{R}^{n}\right)$ if

$$
u=\sum_{j \in \mathbb{Z}} \dot{\Delta}_{j} u \quad \text { in } \mathcal{S}^{\prime}\left(\mathbb{R}^{n}\right)
$$

and

$$
\|u\|_{\dot{B}_{p, 1}^{s}\left(\mathbb{R}^{n}\right)}:=\sum_{j \in \mathbb{Z}} 2^{j s}\left\|\dot{\Delta}_{j} u\right\|_{L^{p}\left(\mathbb{R}^{n}\right)}<\infty .
$$

Here $\left(\dot{\Delta}_{j}\right)_{j \in \mathbb{Z}}$ denotes a homogeneous dyadic resolution of unity in Fourier variables -the socalled Littlewood-Paley decomposition (see e.g. [1], Chap. 2 for more details on the LittlewoodPaley decomposition and Besov spaces).

Loosely speaking, a function belongs to $\dot{B}_{p, 1}^{s}\left(\mathbb{R}^{n}\right)$ if it has $s$ derivatives in $L^{p}\left(\mathbb{R}^{n}\right)$. In the present paper, we shall mainly use the following classical properties:

- the Besov space $\dot{B}_{p, 1}^{n / p}\left(\mathbb{R}^{n}\right)$ is a Banach algebra embedded in the set of continuous functions going to 0 at infinity, whenever $1 \leq p<\infty$;

- the usual product maps $\dot{B}_{p, 1}^{n / p-1}\left(\mathbb{R}^{n}\right) \times \dot{B}_{p, 1}^{n / p}\left(\mathbb{R}^{n}\right)$ in $\dot{B}_{p, 1}^{n / p-1}\left(\mathbb{R}^{n}\right)$ whenever $1 \leq p<2 n$;

- Let $F: I \rightarrow \mathbb{R}$ be a smooth function (with $I$ an open interval of $\mathbb{R}$ containing 0 ) vanishing at 0 . Then for any $s>0,1 \leq p \leq \infty$ and interval $J$ compactly supported in $I$ there exists a constant $C$ such that

$$
\|F(a)\|_{\dot{B}_{p, 1}^{s}\left(\mathbb{R}^{n}\right)} \leq C\|a\|_{\dot{B}_{p, 1}^{s}\left(\mathbb{R}^{n}\right)}
$$

for any $a \in \dot{B}_{p, 1}^{s}\left(\mathbb{R}^{n}\right)$ with values in $J$. In addition, if $a_{1}$ and $a_{2}$ are two such functions and $s=n / p$ then we have

$$
\left\|F\left(a_{2}\right)-F\left(a_{1}\right)\right\|_{\dot{B}_{p, 1}^{n / p}\left(\mathbb{R}^{n}\right)} \leq C\left\|a_{2}-a_{1}\right\|_{\dot{B}_{p, 1}^{n / p}\left(\mathbb{R}^{n}\right)} .
$$

From now on, we shall omit $\mathbb{R}^{n}$ in the notation for Besov spaces. We shall obtain the existence and uniqueness of a local-in-time solution $(\bar{\rho}, \bar{u})$ for $(1.2)$, with $\bar{a}:=\bar{\rho}-1$ in $\mathcal{C}\left([0, T] ; \dot{B}_{p, 1}^{n / p}\right)$ and $\bar{u}$ in the space

$$
E_{p}(T):=\left\{v \in \mathcal{C}\left([0, T] ; \dot{B}_{p, 1}^{n / p-1}\right), \partial_{t} v, \nabla^{2} v \in L^{1}\left(0, T ; \dot{B}_{p, 1}^{n / p-1}\right)\right\} .
$$

That space will be endowed with the norm

$$
\|v\|_{E_{p}(T)}:=\|v\|_{L_{T}^{\infty}\left(\dot{B}_{p, 1}^{n / p-1}\right)}+\left\|\partial_{t} v, \nabla^{2} v\right\|_{L_{T}^{1}\left(\dot{B}_{p, 1}^{n / p-1}\right)} .
$$

Let us now state our main result.

Theorem 1. Let $1<p<2 n$ and $n \geq 2$. Let $u_{0}$ be a vector-field in $\dot{B}_{p, 1}^{n / p-1}$. Assume that the initial density $\rho_{0}$ satisfies $a_{0}:=\left(\rho_{0}-1\right) \in \dot{B}_{p, 1}^{n / p}$ and

$$
\inf _{x} \rho_{0}(x)>0 \text {. }
$$

Then System (1.2) has a unique local solution $(\bar{\rho}, \bar{u})$ with $(\bar{a}, \bar{u}) \in \mathcal{C}\left([0, T] ; \dot{B}_{p, 1}^{n / p}\right) \times E_{p}(T)$. Moreover, the flow map $\left(a_{0}, u_{0}\right) \longmapsto(\bar{a}, \bar{u})$ is Lipschitz continuous from $\dot{B}_{p, 1}^{n / p} \times \dot{B}_{p, 1}^{n / p-1}$ to $\mathcal{C}\left([0, T] ; \dot{B}_{p, 1}^{n / p}\right) \times E_{p}(T)$. 
If $\rho_{0}$ is close enough to some positive constant then the statement holds true for all $p \in[1, \infty)$ and $n \geq 1$.

In Eulerian coordinates, this result recasts in:

Theorem 2. Under the hypotheses of Theorem 1 with $1<p<2 n$ and $n \geq 2$, System (0.1) has a unique local solution $(\rho, u)$ with $u \in E_{p}(T), \rho$ bounded away from 0 and $(\rho-1) \in \mathcal{C}\left([0, T] ; \dot{B}_{p, 1}^{n / p}\right)$.

Let us make a few comments concerning the above assumptions.

- We expect the Lagrangian method to improve the uniqueness conditions given in e.g. [8] for the full Navier-Stokes equations. We here consider the barotropic case for simplicity.

- The condition $1 \leq p<2 n$ is a consequence of the product laws in Besov spaces. It implies that the regularity exponent for the velocity has to be greater than $-1 / 2$ (to be compared with -1 for the homogeneous incompressible Navier-Stokes equations). It would be interesting to see whether introducing a modified velocity as in B. Haspot's works $[16,17]$ allows to consider different Lebesgue exponents for the Besov spaces pertaining to the density and the velocity so as to go beyond $p=2 n$ for the velocity.

- The regularity condition over the density is stronger than that for density-dependent incompressible fluids (see [14]). In particular, in contrast with incompressible fluids, it is not clear that combining Lagrangian coordinates and critical regularity approach allows to consider discontinuous densities.

- Owing to the fact that the density satisfies a transport equation, we do not expect Lipschitz continuity of the flow map in high norm for the Eulerian formulation to be true.

- It is worth comparing our results with those of P. Germain in [15], and D. Hoff in [18] concerning the weak-strong uniqueness problem. In both papers, the idea is to show that, in the constant viscosity case, a finite energy weak solution coincides with a strong one under some additional assumptions. The weak solution turns out to have less regularity than in Theorem 2. At the same time, the assumptions on the strong solution $(\rho, u)$ are much stronger. In both papers, $\nabla u$ has to be in $L^{1}\left(0, T ; L^{\infty}\right)$, and to satisfy additional conditions: roughly $\nabla^{2} u$ or $\partial_{t} u$ have to be in $L^{2}\left(0, T ; L^{d}\right)$ in Germain's work, while $\sqrt{t} D^{2} u \in L^{r}\left(0, T ; L^{4}\right)$ with $r=4 / 3$ if $n=2$, and $r=8 / 5$ if $n=3$ in Hoff's paper. Some regularity conditions are required on the density but they are, to some extent, weaker than ours.

\section{The simple case of almost homogeneous Compressible Fluids}

As a warm up and for the reader convenience, we here explain how local well-posedness may be proved for the system in Lagrangian coordinates in the simple case where:

(1) The viscosity coefficients are constant,

(2) The density is very close to one.

Let $\mu^{\prime}:=\lambda+\mu$. Keeping in mind the above two conditions and using the fact that the first equation of (1.2) implies that

$$
J(t, \cdot) \bar{\rho}(t, \cdot) \equiv \rho_{0},
$$

with $J:=|\operatorname{det} D X|$ and

$$
X(t, y):=y+\int_{0}^{t} \bar{u}(\tau, y) d \tau,
$$

we rewrite the equation for the Lagrangian velocity as (recall that $\left.A:=(D X)^{-1}\right)$ :

$$
\begin{aligned}
\partial_{t} \bar{u}-\mu \Delta \bar{u}-\mu^{\prime} \nabla \operatorname{div} \bar{u} & =\left(1-\rho_{0}\right) \partial_{t} \bar{u}+2 \mu \operatorname{div}\left(\operatorname{adj}(D X) D_{A}(\bar{u})-D(\bar{u})\right) \\
& +\lambda \operatorname{div}\left(\operatorname{adj}(D X) \operatorname{div}_{A} \bar{u}-\operatorname{div} \bar{u} \text { Id }\right)-\operatorname{div}\left(\operatorname{adj}(D X) P\left(J^{-1} \rho_{0}\right)\right) .
\end{aligned}
$$


The left-hand side of the above equation is the linear Lamé system with constant coefficients, the solvability of which may be easily deduced from that of the heat equation in the whole space (see e.g. [1], Chap. 2 or [13]). We get:

Proposition 1. Let the viscosity coefficients $\left(\mu, \mu^{\prime}\right) \in \mathbb{R}^{2}$ satisfy $\mu>0$ and $\mu+\mu^{\prime}>0$. Let $p \in[1, \infty]$ and $s \in \mathbb{R}$. Let $u_{0} \in \dot{B}_{p, 1}^{s}$ and $f \in L^{1}\left(0, T ; \dot{B}_{p, 1}^{s}\right)$. Then the Lamé system

$$
\left\{\begin{array}{llr}
\partial_{t} u-\mu \Delta u-\mu^{\prime} \nabla \operatorname{div} u=f & \text { in } & (0, T) \times \mathbb{R}^{n} \\
\left.u\right|_{t=t_{0}}=u_{0} & \text { on } & \mathbb{R}^{n}
\end{array}\right.
$$

has a unique solution $u$ in $\mathcal{C}\left([0, T) ; \dot{B}_{p, 1}^{s}\right)$ such that $\partial_{t} u, \nabla^{2} u \in L^{1}\left(0, T ; \dot{B}_{p, 1}^{s}\right)$ and the following estimate is valid:

$$
\|u\|_{L_{T}^{\infty}\left(\dot{B}_{p, 1}^{s}\right)}+\min \left(\mu, \mu+\mu^{\prime}\right)\left\|\nabla^{2} u\right\|_{L_{T}^{1}\left(\dot{B}_{p, 1}^{s}\right)} \leq C\left(\|f\|_{L_{T}^{1}\left(\dot{B}_{p, 1}^{s}\right)}+\left\|u_{0}\right\|_{\dot{B}_{p, 1}^{s}}\right)
$$

where $C$ is an absolute constant with no dependence on $\mu, \mu^{\prime}$ and $T$.

In the rest of this section, we drop the bars on the Lagrangian velocity field. Granted with the above proposition, we define a map $\Phi: v \mapsto u$ on $E_{p}(T)$ where $u$ stands for the solution to

$$
\partial_{t} u-\mu \Delta u-\mu^{\prime} \nabla \operatorname{div} u=I_{1}(v)+2 \mu \operatorname{div} I_{2}(v, v)+\lambda \operatorname{div} I_{3}(v, v)-\operatorname{div} I_{4}(v)
$$

with initial data $u_{0}$ and

$$
\begin{aligned}
& I_{1}(w)=-a_{0} \partial_{t} w, \quad I_{2}(v, w)=\operatorname{adj}\left(D X_{v}\right) D_{A_{v}}(w)-D(w), \\
& I_{3}(v, w)=\operatorname{div}_{A_{v}} w \operatorname{adj}\left(D X_{v}\right)-\operatorname{div} w \mathrm{Id}, \quad I_{4}(v)=\operatorname{adj}\left(D X_{v}\right) P\left(J_{v}^{-1} \rho_{0}\right) .
\end{aligned}
$$

Note that any fixed point of $\Phi$ is a solution in $E_{p}(T)$ to (2.3). We claim that the existence of such points is a consequence of the standard Banach fixed point theorem in a suitable closed ball of $E_{p}(T)$.

First step: estimates for $I_{1}, I_{2}, I_{3}$ and $I_{4}$. Throughout we assume that for a small enough constant $c$,

$$
\int_{0}^{T}\|D v\|_{\dot{B}_{p, 1}^{n / p}} d t \leq c
$$

It is obvious that

$$
\left\|I_{1}(w)\right\|_{L_{T}^{1}\left(\dot{B}_{p, 1}^{n / p-1}\right)} \leq\left\|a_{0}\right\|_{\mathcal{M}\left(\dot{B}_{p, 1}^{n / p-1}\right)}\left\|\partial_{t} w\right\|_{L_{T}^{1}\left(\dot{B}_{p, 1}^{n / p-1}\right)}
$$

where the multiplier norm $\mathcal{M}\left(\dot{B}_{p, 1}^{s}\right)$ for $\dot{B}_{p, 1}^{s}$, is defined by

$$
\|f\|_{\mathcal{M}\left(\dot{B}_{p, 1}^{s}\right)}:=\sup \|\psi f\|_{\dot{B}_{p, 1}^{s}} .
$$

The supremum is taken over those functions $\psi$ in $\dot{B}_{p, 1}^{s}$ with norm 1.

Next, taking advantage of the fact that $\dot{B}_{p, 1}^{n / p}$ is an algebra if $1 \leq p<\infty$, of (A.12), (A.13) and (2.7), we readily get

$$
\left\|I_{2}(v, w)\right\|_{L_{T}^{1}\left(\dot{B}_{p, 1}^{n / p}\right)}+\left\|I_{3}(v, w)\right\|_{L_{T}^{1}\left(\dot{B}_{p, 1}^{n / p}\right)} \leq C\|D v\|_{L_{T}^{1}\left(\dot{B}_{p, 1}^{n / p}\right)}\|D w\|_{L_{T}^{1}\left(\dot{B}_{p, 1}^{n / p}\right)} .
$$

As regards the pressure term (that is $I_{4}(v)$ ), we use the fact that under assumption (2.7), we have, by virtue of the composition inequality (1.5) and of flow estimates (see (A.9) and (A.11)),

$$
\left\|I_{4}(v)\right\|_{L_{T}^{\infty}\left(\dot{B}_{p, 1}^{n / p}\right)} \leq C\left(1+\|D v\|_{L_{T}^{1}\left(\dot{B}_{p, 1}^{n / p}\right)}\right)\left(1+\left\|a_{0}\right\|_{\dot{B}_{p, 1}^{n / p}}\right) .
$$


Second step: $\Phi$ maps a suitable closed ball in itself. At this stage, one may assert that if $v \in E_{p}(T)$ satisfies $(2.7)$ then the right-hand side of $(2.6)$ belongs to $L^{1}\left(0, T ; \dot{B}_{p, 1}^{n / p-1}\right)$. Hence Proposition 1 implies that $\Phi(v)$ is well defined and maps $E_{p}(T)$ to itself. However it is not clear that it is contractive over the whole set $E_{p}(T)$. So we introduce $u_{L}$ the "free solution" to

$$
\partial_{t} u_{L}-\mu \Delta u_{L}-\mu^{\prime} \nabla \operatorname{div} u_{L}=0,\left.\quad u_{L}\right|_{t=0}=u_{0} .
$$

Proposition 1 guarantees that $u_{L}$ belongs to $E_{p}(T)$ for all $T>0$.

We claim that if $T$ is small enough (a condition which will be expressed in terms of the free solution $u_{L}$ ) and if $R$ is small enough (a condition which will depend only on the viscosity coefficients and on $p, n$ and $P$ ) then $v \in \bar{B}_{E_{p}(T)}\left(u_{L}, R\right)$ implies that (2.7) is fulfilled and that $u \in \bar{B}_{E_{p}(T)}\left(u_{L}, R\right)$. Indeed $\widetilde{u}:=u-u_{L}$ satisfies

$$
\left\{\begin{array}{l}
\partial_{t} \widetilde{u}-\mu \Delta \widetilde{u}-\mu^{\prime} \nabla \operatorname{div} \widetilde{u}=I_{1}(v)+2 \mu \operatorname{div} I_{2}(v, v)+\lambda \operatorname{div} I_{3}(v, v)-\operatorname{div} I_{4}(v), \\
\left.\widetilde{u}\right|_{t=0}=0 .
\end{array}\right.
$$

So Proposition 1 yields $^{1}$

$$
\|\widetilde{u}\|_{E_{p}(T)} \lesssim\left\|I_{1}(v)\right\|_{L_{T}^{1}\left(\dot{B}_{p, 1}^{n / p-1}\right)}+\left\|I_{2}(v, v)\right\|_{L_{T}^{1}\left(\dot{B}_{p, 1}^{n / p}\right)}+\left\|I_{3}(v, v)\right\|_{L_{T}^{1}\left(\dot{B}_{p, 1}^{n / p}\right)}+T\left\|I_{4}(v)\right\|_{L_{T}^{\infty}\left(\dot{B}_{p, 1}^{n / p}\right)} .
$$

Inserting inequalities (2.8), (2.10) and (2.11), we thus get:

$$
\|\widetilde{u}\|_{E_{p}(T)} \lesssim\|D v\|_{L_{T}^{1}\left(\dot{B}_{p, 1}^{n / p}\right)}^{2}+\left\|a_{0}\right\|_{\mathcal{M}\left(\dot{B}_{p, 1}^{n / p-1}\right)}\left\|\partial_{t} v\right\|_{L_{T}^{1}\left(\dot{B}_{p, 1}^{n / p-1}\right)}+T\left(1+\left\|a_{0}\right\|_{\dot{B}_{p, 1}^{n / p}}\right) .
$$

That is, keeping in mind that $v$ is in $\bar{B}_{E_{p}(T)}\left(u_{L}, R\right)$,

$$
\|\widetilde{u}\|_{E_{p}(T)} \leq C\left(\left\|a_{0}\right\|_{\mathcal{M}\left(\dot{B}_{p, 1}^{n / p-1}\right)}\left(R+\left\|\partial_{t} u_{L}\right\|_{L_{T}^{1}\left(\dot{B}_{p, 1}^{n / p-1}\right)}\right)+\left\|D u_{L}\right\|_{L_{T}^{1}\left(\dot{B}_{p, 1}^{n / p}\right)}^{2}+R^{2}+T\left(1+\left\|a_{0}\right\|_{\dot{B}_{p, 1}^{n / p}}\right)\right) .
$$

So we see that if $T$ satisfies

$$
C T\left(1+\left\|a_{0}\right\|_{\dot{B}_{p, 1}^{n / p}}\right) \leq R / 2 \quad \text { and } \quad\left\|D u_{L}\right\|_{L_{T}^{1}\left(\dot{B}_{p, 1}^{n / p}\right)}+\left\|\partial_{t} u_{L}\right\|_{L_{T}^{1}\left(\dot{B}_{p, 1}^{n / p-1}\right)} \leq R
$$

then we have

$$
\|\widetilde{u}\|_{E_{p}(T)} \leq 2 C\left\|a_{0}\right\|_{\mathcal{M}\left(\dot{B}_{p, 1}^{n / p-1}\right)} R+2 C R^{2}+R / 2 .
$$

Hence there exists a small constant $\eta=\eta(n, p)$ such that if

$$
\left\|a_{0}\right\|_{\mathcal{M}\left(\dot{B}_{p, 1}^{n / p-1}\right)} \leq \eta
$$

and if $R$ has been chosen small enough then $u$ is in $\bar{B}_{E_{p}(T)}\left(u_{L}, R\right)$. Of course, taking $R$ and $T$ even smaller ensures that $(2.7)$ is satisfied for all vector-field of $\bar{B}_{E_{p}(T)}\left(u_{L}, R\right)$.

Third step: contraction properties. We claim that under Conditions (2.13) and (2.12) (with a smaller $R$ if needed), the map $\Phi$ is $1 / 2$-Lipschitz over $\bar{B}_{E_{p}(T)}\left(u_{L}, R\right)$. So we are given $v_{1}$ and $v_{2}$ in $\bar{B}_{E_{p}(T)}\left(u_{L}, R\right)$ and denote

$$
u_{1}:=\Phi\left(v_{1}\right) \quad \text { and } \quad u_{2}:=\Phi\left(v_{2}\right) .
$$

Let $X_{1}$ and $X_{2}$ be the flows associated to $v_{1}$ and $v_{2}$. Set $A_{i}=\left(D X_{i}\right)^{-1}$ and $J_{i}:=\operatorname{det} D X_{i}$ for $i=1,2$. The equation satisfied by $\delta u:=u_{2}-u_{1}$ reads

$$
\partial_{t} \delta u-\mu \Delta \delta u-\mu^{\prime} \nabla \operatorname{div} \delta u=\delta f:=\delta f_{1}+\operatorname{div} \delta f_{2}+2 \mu \operatorname{div} \delta f_{3}+\lambda \operatorname{div} \delta f_{4}
$$

\footnotetext{
${ }^{1}$ For simplicity, we do not track the dependency of the coefficients with respect to $\mu$ and $\mu^{\prime}$.
} 
with $\delta f_{1}:=-a_{0} \partial_{t} \delta u$,

$$
\begin{gathered}
\delta f_{2}:=\operatorname{adj}\left(D X_{1}\right) P\left(\rho_{0} J_{1}^{-1}\right)-\operatorname{adj}\left(D X_{2}\right) P\left(\rho_{0} J_{2}^{-1}\right), \\
\delta f_{3}:=\operatorname{adj}\left(D X_{2}\right) D_{A_{2}}\left(u_{2}\right)-\operatorname{adj}\left(D X_{1}\right) D_{A_{1}}\left(u_{1}\right)-D(\delta u), \\
\delta f_{4}:=\operatorname{adj}\left(D X_{2}\right)^{T} A_{2}: \nabla u_{2}-\operatorname{adj}\left(D X_{1}\right)^{T} A_{1}: \nabla u_{1}-\operatorname{div} \delta u \text { Id. }
\end{gathered}
$$

Once again, bounding $\delta u$ in $E_{p}(T)$ stems from Proposition 1, which ensures that

$$
\|\delta u\|_{E_{p}(T)} \lesssim\left\|\delta f_{1}\right\|_{L_{T}^{1}\left(\dot{B}_{p, 1}^{n / p-1}\right)}+T\left\|\delta f_{2}\right\|_{L_{T}^{\infty}\left(\dot{B}_{p, 1}^{n / p}\right)}+\left\|\delta f_{3}\right\|_{L_{T}^{1}\left(\dot{B}_{p, 1}^{n / p}\right)}+\left\|\delta f_{4}\right\|_{L_{T}^{1}\left(\dot{B}_{p, 1}^{n / p}\right)} .
$$

In order to bound $\delta f_{1}$, we just have to use the definition of the multiplier space $\mathcal{M}\left(\dot{B}_{p, 1}^{n / p-1}\right)$. We get

$$
\left\|\delta f_{1}\right\|_{L_{T}^{1}\left(\dot{B}_{p, 1}^{n / p-1}\right)} \leq\left\|a_{0}\right\|_{\mathcal{M}\left(\dot{B}_{p, 1}^{n / p-1}\right)}\left\|\partial_{t} \delta u\right\|_{L_{T}^{1}\left(\dot{B}_{p, 1}^{n / p-1}\right)} .
$$

Next, using the decomposition

$$
\delta f_{2}=\left(\operatorname{adj}\left(D X_{1}\right)-\operatorname{adj}\left(D X_{2}\right)\right) P\left(\rho_{0} J_{2}^{-1}\right)+\operatorname{adj}\left(D X_{1}\right)\left(P\left(\rho_{0} J_{1}^{-1}\right)-P\left(\rho_{0} J_{2}^{-1}\right)\right),
$$

together with composition inequalities (1.5), (1.6) and (A.19), and product laws in Besov space yields

$$
\left\|\delta f_{2}\right\|_{L_{T}^{\infty}\left(\dot{B}_{p, 1}^{n / p}\right)} \lesssim T\left(1+\left\|a_{0}\right\|_{\dot{B}_{p, 1}^{n / p}}\right)\|D \delta v\|_{L_{T}^{1}\left(\dot{B}_{p, 1}^{n / p}\right)}
$$

Finally, we have

$\delta f_{4}=\left(\operatorname{adj}\left(D X_{2}\right)-\operatorname{adj}\left(D X_{1}\right)\right)^{T} A_{2}: \nabla u_{2}+\operatorname{adj}\left(D X_{1}\right)^{T}\left(A_{2}-A_{1}\right): \nabla u_{2}+\left(\operatorname{adj}\left(D X_{1}\right)^{T} A_{1}-\mathrm{Id}\right): \nabla \delta u$, whence, by virtue of (A.9), (A.10), (A.18) and (A.19),

$$
\left\|\delta f_{4}\right\|_{L_{T}^{1}\left(\dot{B}_{p, 1}^{n / p}\right)} \lesssim\|D \delta v\|_{L_{T}^{1}\left(\dot{B}_{p, 1}^{n / p}\right)}\left\|D u_{2}\right\|_{L_{T}^{1}\left(\dot{B}_{p, 1}^{n / p}\right)}+\|D \delta u\|_{L_{T}^{1}\left(\dot{B}_{p, 1}^{n / p}\right)}\left\|D v_{1}\right\|_{L_{T}^{1}\left(\dot{B}_{p, 1}^{n / p}\right)} .
$$

Bounding $\delta f_{3}$ works exactly the same. So we see that if Conditions (2.12) and (2.13) are satisfied (with smaller $\eta$ and larger $C$ if need be) then we have

$$
\|\delta u\|_{E_{p}(T)} \leq \frac{1}{2}\|\delta v\|_{E_{p}(T)} .
$$

Hence, the map $\Phi: \bar{B}_{E_{p}(T)}\left(u_{L}, R\right) \mapsto \bar{B}_{E_{p}(T)}\left(u_{L}, R\right)$ is $1 / 2$-Lipschitz. Therefore, Banach' fixed point theorem ensures that $\Phi$ admits a unique fixed point in $\bar{B}_{E_{p}(T)}\left(u_{L}, R\right)$. This completes the proof of existence of a solution in $E_{p}(T)$ for System (1.2).

A tiny variation over the proof of the contraction properties yields uniqueness and Lipschitz continuity of the flow map. We eventually get:

Theorem 3. Assume that $n \geq 1$. Let $p \in[1, \infty)$ and $u_{0}$ be a vector-field in $\dot{B}_{p, 1}^{n / p-1}$. Assume that the initial density $\rho_{0}$ satisfies $a_{0}:=\left(\rho_{0}-1\right) \in \dot{B}_{p, 1}^{n / p}$. There exists a constant $c$ depending only on $p$ and on $n$ such that if

$$
\left\|a_{0}\right\|_{\mathcal{M}\left(\dot{B}_{p, 1}^{n / p-1}\right)} \leq c
$$

then System (1.2) has a unique local solution $(\bar{\rho}, \bar{u})$ with $(\bar{a}, \bar{u}) \in \mathcal{C}\left([0, T] ; \dot{B}_{p, 1}^{n / p}\right) \times E_{p}(T)$. Moreover, the flow map $\left(a_{0}, u_{0}\right) \longmapsto(\bar{a}, \bar{u})$ is Lipschitz continuous from $B_{p, 1}^{n / p} \times \dot{B}_{p, 1}^{n / p-1}$ to $\mathcal{C}\left([0, T] ; \dot{B}_{p, 1}^{n / p}\right) \times E_{p}(T)$.

In Eulerian coordinates, this result recasts in:

Theorem 4. Under the above assumptions with in addition $n \geq 2$ and $p<2 n$, System (0.1) has a unique local solution $(\rho, u)$ with density bounded away from vacuum and $a \in \mathcal{C}\left([0, T] ; \dot{B}_{p, 1}^{n / p-1}\right)$ and $u \in E_{p}(T)$. 
We do not give here more details on how to complete the proof of Theorem 3 and its Eulerian counterpart, Theorem 4, as it will done in the next section under much more general assumptions.

\section{The fully nonhomogeneous Case}

For treating the general case where $\rho_{0}$ only satisfies (1.7), just resorting to Proposition 1 is not enough because the term $I_{1}(v, v)$ in the r.h.s. of $(2.6)$ need not be small. One has first to establish a similar statement for a Lamé system with nonconstant coefficients. More precisely, keeping in mind that $\rho=J_{u}^{-1} \rho_{0}$ (we still drop the bars for notational simplicity), we recast the velocity equation of (1.2) in:

$$
L_{\rho_{0}}(u)=\rho_{0}^{-1} \operatorname{div}\left(I_{1}(u, u)+I_{2}(u, u)+I_{3}(u, u)+I_{4}(u)\right)
$$

with

$$
L_{\rho_{0}}(u):=\partial_{t} u-\rho_{0}^{-1} \operatorname{div}\left(2 \mu\left(\rho_{0}\right) D(u)+\lambda\left(\rho_{0}\right) \operatorname{div} u \mathrm{Id}\right)
$$

and

$$
\begin{aligned}
& I_{1}(v, w):=\left(\operatorname{adj}\left(D X_{v}\right)-\mathrm{Id}\right)\left(\mu\left(J_{v}^{-1} \rho_{0}\right)\left(D w A_{v}+{ }^{T} A_{v} \nabla w\right)+\lambda\left(J_{v}^{-1} \rho_{0}\right)\left({ }^{T} A_{v}: \nabla w\right) \operatorname{Id}\right) \\
& I_{2}(v, w):=\left(\mu\left(J_{v}^{-1} \rho_{0}\right)-\mu\left(\rho_{0}\right)\right)\left(D w A_{v}+{ }^{T} A_{v} \nabla w\right)+\left(\lambda\left(J_{v}^{-1} \rho_{0}\right)-\lambda\left(\rho_{0}\right)\right)\left({ }^{T} A_{v}: \nabla w\right) \operatorname{Id} \\
& I_{3}(v, w):=\mu\left(\rho_{0}\right)\left(D w\left(A_{v}-\mathrm{Id}\right)+{ }^{T}\left(A_{v}-\mathrm{Id}\right) \nabla w\right)+\lambda\left(\rho_{0}\right)\left({ }^{T}\left(A_{v}-\mathrm{Id}\right): \nabla w\right) \operatorname{Id} \\
& I_{4}(v):=-\operatorname{adj}\left(D X_{v}\right) P\left(\rho_{0} J_{v}^{-1}\right) .
\end{aligned}
$$

Therefore, in order to solve (1.2) locally, it suffices to show that the map

$$
\Phi: v \longmapsto u
$$

with $u$ the solution to

$$
\left\{\begin{array}{l}
L_{\rho_{0}}(u)=\rho_{0}^{-1} \operatorname{div}\left(I_{2}(v, v)+I_{3}(v, v)+I_{4}(v, v)+I_{5}(v)\right), \\
\left.u\right|_{t=0}=u_{0}
\end{array}\right.
$$

has a fixed point in $E_{p}(T)$ for small enough $T$.

As a first step, we have to study the properties of the linear Lamé operator $L_{\rho_{0}}$. This is done in the following subsection.

3.1. Linear parabolic systems with rough coefficients. As a warm up, we consider the following scalar heat equation with variable coefficients:

$$
\partial_{t} u-a \operatorname{div}(b \nabla u)=f .
$$

We assume that

$$
\alpha:=\inf _{(t, x) \in[0, T] \times \mathbb{R}^{n}}(a b)(t, x)>0 .
$$

Let us first consider the smooth case.

Proposition 2. Assume that $a$ and $b$ are bounded functions satisfying (3.4) and such that $b \nabla a$ and $a \nabla b$ are in $L^{2}\left(0, T ; \dot{B}_{p, 1}^{n / p}\right)$ for some $1<p<\infty$. There exist two constants $\kappa=\kappa(p)$ and $C=C(s, n, p)$ such that the solutions to (3.3) satisfy for all $t \in[0, T]$,

$$
\|u\|_{L_{t}^{\infty}\left(\dot{B}_{p, 1}^{s}\right)}+\kappa \alpha\|u\|_{L_{t}^{1}\left(\dot{B}_{p, 1}^{s+2}\right)} \leq\left(\left\|u_{0}\right\|_{\dot{B}_{p, 1}^{s}}+\|f\|_{L_{t}^{1}\left(\dot{B}_{p, 1}^{s}\right)}\right) \exp \left(\frac{C}{\alpha} \int_{0}^{t}\|(b \nabla a, a \nabla b)\|_{\dot{B}_{p, 1}^{n / p}}^{2} d \tau\right)
$$

whenever $-\min \left(n / p, n / p^{\prime}\right)<s \leq n / p$. 
Proof. We first rewrite the equation for $u$ as follows:

$$
\partial_{t} u-\operatorname{div}(a b \nabla u)=f-b \nabla a \cdot \nabla u
$$

then localize the equation in the Fourier space, according to Littlewood-Paley decomposition:

$$
\partial_{t} u_{j}-\operatorname{div}\left(a b \nabla u_{j}\right)=f_{j}-\dot{\Delta}_{j}(b \nabla a \cdot \nabla u)+R_{j}
$$

with $u_{j}:=\dot{\Delta}_{j} u, f_{j}:=\dot{\Delta}_{j} f$ and $R_{j}:=\operatorname{div}\left(\left[\dot{\Delta}_{j}, a b\right] \nabla u\right)$.

Next, we multiply the above equation by $u_{j}\left|u_{j}\right|^{p-2}$ and integrate over $\mathbb{R}^{n}$. Taking advantage of Lemma 8 in the appendix of [12] (here $1<p<\infty$ comes into play) and of Hölder inequality, we get for some constant $c_{p}$ depending only on $p$ :

$$
\frac{1}{p} \frac{d}{d t}\left\|u_{j}\right\|_{L^{p}}^{p}+c_{p} \alpha 2^{2 j}\left\|u_{j}\right\|_{L^{p}}^{p} \leq\left\|u_{j}\right\|_{L^{p}}^{p-1}\left(\left\|f_{j}\right\|_{L^{p}}+\left\|\dot{\Delta}_{j}(b \nabla a \cdot \nabla u)\right\|_{L^{p}}+\left\|R_{j}\right\|_{L^{p}}\right),
$$

which, after time integration, leads to

$$
\begin{aligned}
\left\|u_{j}\right\|_{L_{t}^{\infty}\left(L^{p}\right)}+c_{p} \alpha 2^{2 j}\left\|u_{j}\right\|_{L_{t}^{1}\left(L^{p}\right)} \leq & \left\|u_{0, j}\right\|_{L^{p}} \\
& +\left\|f_{j}\right\|_{L_{t}^{1}\left(L^{p}\right)}+\int_{0}^{t}\left(\left\|\dot{\Delta}_{j}(b \nabla a \cdot \nabla u)\right\|_{L^{p}}+\left\|R_{j}\right\|_{L^{p}}\right) d \tau
\end{aligned}
$$

According to Lemmas 4 and 5 in Appendix, there exist a positive constant $C$ and some sequence $\left(c_{j}\right)_{j \in \mathbb{Z}}$ with $\|c\|_{\ell^{1}(\mathbb{Z})}=1$, satisfying

$$
\left\|\dot{\Delta}_{j}(b \nabla a \cdot \nabla u)\right\|_{L^{p}}+\left\|R_{j}\right\|_{L^{p}} \leq C c_{j} 2^{-j s}\left(\|b \nabla a\|_{\dot{B}_{p, 1}^{n / p}}+\|a \nabla b\|_{\dot{B}_{p, 1}^{n / p}}\right)\|\nabla u\|_{\dot{B}_{p, 1}^{s}} .
$$

Then inserting (3.6) in (3.5), multiplying by $2^{j s}$ and summing up over $j$ yields

$$
\begin{aligned}
\|u\|_{L_{t}^{\infty}\left(\dot{B}_{p, 1}^{s}\right)}+c_{p} \alpha\|u\|_{L_{t}^{1}\left(\dot{B}_{p, 1}^{s+2}\right)} \leq\left\|u_{0}\right\|_{\dot{B}_{p, 1}^{s}}+\|f\|_{L_{t}^{1}\left(\dot{B}_{p, 1}^{s}\right)} & \\
& +C \int_{0}^{t}\|(b \nabla a, a \nabla b)\|_{\dot{B}_{p, 1}^{n / p}}\|u\|_{\dot{B}_{p, 1}^{s+1}} d \tau .
\end{aligned}
$$

From the interpolation inequality

$$
\|u\|_{\dot{B}_{p, 1}^{s+1}} \leq\|u\|_{\dot{B}_{p, 1}^{s}}^{1 / 2}\|u\|_{\dot{B}_{p, 1}^{s+2}}^{1 / 2}
$$

we gather that

$$
C\|(b \nabla a, a \nabla b)\|_{\dot{B}_{p, 1}^{n / p}}\|u\|_{\dot{B}_{p, 1}^{s+1}} \leq \frac{\alpha c_{p}}{2}\|u\|_{\dot{B}_{p, 1}^{s+2}}+\frac{C^{2}}{2 \alpha c_{p}}\|(b \nabla a, a \nabla b)\|_{\dot{B}_{p, 1}^{n / p}}^{2}\|u\|_{\dot{B}_{p, 1}^{s}} .
$$

So plugging this in (3.7) and applying Gronwall lemma completes the proof of the proposition.

In the rough case where the coefficients are only in $\dot{B}_{p, 1}^{n / p}$, the above proposition has to be modified as follows:

Proposition 3. Let $a$ and $b$ be bounded positive and satisfy (3.4). Assume that $b \nabla a$ and $a \nabla b$ are in $L^{\infty}\left(0, T ; \dot{B}_{p, 1}^{n / p-1}\right)$ with $1<p<\infty$. There exist three constants $\eta, \kappa$ and $C$ such that if for some $m \in \mathbb{Z}$ we have

$$
\begin{gathered}
\inf _{(t, x) \in[0, T] \times \mathbb{R}^{n}} \dot{S}_{m}(a b)(t, x) \geq \alpha / 2, \\
\left\|\left(\operatorname{Id}-\dot{S}_{m}\right)(b \nabla a, a \nabla b)\right\|_{L_{T}^{\infty}\left(\dot{B}_{p, 1}^{n / p-1}\right)} \leq \eta \alpha
\end{gathered}
$$

then the solution to (3.3) satisfies for all $t \in[0, T]$,

$$
\|u\|_{L_{t}^{\infty}\left(\dot{B}_{p, 1}^{s}\right)}+\alpha \kappa\|u\|_{L_{t}^{1}\left(\dot{B}_{p, 1}^{s+2}\right)} \leq\left(\left\|u_{0}\right\|_{\dot{B}_{p, 1}^{s}}+\|f\|_{L_{t}^{1}\left(\dot{B}_{p, 1}^{s}\right)}\right) \exp \left(\frac{C}{\alpha} \int_{0}^{t}\left\|\dot{S}_{m}(b \nabla a, a \nabla b)\right\|_{\dot{B}_{p, 1}^{n, p}}^{2} d \tau\right)
$$


whenever

$$
-\min \left(n / p, n / p^{\prime}\right)<s \leq n / p-1 .
$$

Proof. Given the new assumptions, it is natural to replace (3.6) by the inequality

$$
\left\|\dot{\Delta}_{j}(b \nabla a \cdot \nabla u)\right\|_{L^{p}}+\left\|R_{j}\right\|_{L^{p}} \leq C c_{j} 2^{-j s}\left(\|b \nabla a\|_{\dot{B}_{p, 1}^{n / p-1}}+\|a \nabla b\|_{\dot{B}_{p, 1}^{n / p-1}}\right)\|\nabla u\|_{\dot{B}_{p, 1}^{s+1}},
$$

which may be obtained by taking $\sigma=1$ and $\nu=1$ in Lemmas 4 and 5. However, when bounding $R_{j}$, in addition to (3.11), one has to assume that $p \leq n$. Also, as it involves the highest regularity of $u$, we cannot expect to absorb this "remainder term" any longer, unless $a \nabla b$ and $b \nabla a$ are small in $\dot{B}_{p, 1}^{n / p-1}$ (which would correspond to the case that has been treated in the previous section). So we rather rewrite the heat equation as follows:

$$
\partial_{t} u-\operatorname{div}\left(\dot{S}_{m}(a b) \nabla u\right)=f+\operatorname{div}\left(\left(\operatorname{Id}-\dot{S}_{m}\right)(a b) \nabla u\right)-\dot{S}_{m}(b \nabla a) \cdot \nabla u-\left(\operatorname{Id}-\dot{S}_{m}\right)(b \nabla a) \cdot \nabla u .
$$

Now, using the infimum bound for $\dot{S}_{m}(a b)$ and arguing as for proving (3.5), we get

$$
\begin{aligned}
& \left\|u_{j}\right\|_{L_{t}^{\infty}\left(L^{p}\right)}+c_{p} \alpha 2^{2 j}\left\|u_{j}\right\|_{L_{t}^{1}\left(L^{p}\right)} \leq\left\|u_{0, j}\right\|_{L^{p}}+\left\|f_{j}\right\|_{L_{t}^{1}\left(L^{p}\right)}+\int_{0}^{t}\left\|\dot{\Delta}_{j} \operatorname{div}\left(\left(\operatorname{Id}-\dot{S}_{m}\right)(a b) \nabla u\right)\right\|_{L^{p}} d \tau \\
& +\int_{0}^{t}\left(\left\|\dot{\Delta}_{j}\left(\dot{S}_{m}(b \nabla a) \cdot \nabla u\right)\right\|_{L^{p}}+\left\|\dot{\Delta}_{j}\left(\left(\operatorname{Id}-\dot{S}_{m}\right)(b \nabla a) \cdot \nabla u\right)\right\|_{L^{p}}+\left\|\operatorname{div}\left(\left[\dot{S}_{m}(a b), \dot{\Delta}_{j}\right] \nabla u\right)\right\|_{L^{p}}\right) d \tau .
\end{aligned}
$$

The idea is to apply the procedure of the "smooth" case for the low frequency part of the coefficients (that is the part containing $\dot{S}_{m}$ ) and the "perturbation" approach for the other part. More precisely, appealing to Lemmas 4 and 5, we get under Condition (3.11) and for some sequence $\left(c_{j}\right)_{j \in \mathbb{Z}}$ with $\|c\|_{\ell^{1}(\mathbb{Z})}=1$ :

$$
\begin{aligned}
\left\|\dot{\Delta}_{j} \operatorname{div}\left(\left(\operatorname{Id}-\dot{S}_{m}\right)(a b) \nabla u\right)\right\|_{L^{p}} & \lesssim c_{j} 2^{-j s}\left\|\left(\operatorname{Id}-\dot{S}_{m}\right)(a b)\right\|_{\dot{B}_{p, 1}^{n / p}}\|\nabla u\|_{\dot{B}_{p, 1}^{s+1}}, \\
\left\|\dot{\Delta}_{j}\left(\dot{S}_{m}(b \nabla a) \cdot \nabla u\right)\right\|_{L^{p}} & \lesssim c_{j} 2^{-j s}\left\|\dot{S}_{m}(b \nabla a)\right\|_{\dot{B}_{p, p}^{n / p}}\|\nabla u\|_{\dot{B}_{p, 1}^{s}}, \\
\left\|\dot{\Delta}_{j}\left(\left(\operatorname{Id}-\dot{S}_{m}\right)(b \nabla a) \cdot \nabla u\right)\right\|_{L^{p}} & \lesssim c_{j} 2^{-j s}\left\|\left(\operatorname{Id}-\dot{S}_{m}\right)(b \nabla a)\right\|_{\dot{B}_{p, 1}^{n / p-1}}\|\nabla u\|_{\dot{B}_{p, 1}^{s+1}}, \\
\left\|\operatorname{div}\left(\left[\dot{S}_{m}(a b), \dot{\Delta}_{j}\right] \nabla u\right)\right\|_{L^{p}} & \lesssim c_{j} 2^{-j s}\left\|\dot{S}_{m} \nabla(a b)\right\|_{\dot{B}_{p, 1}^{n / p}}\|\nabla u\|_{\dot{B}_{p, 1}^{s}} .
\end{aligned}
$$

Let us plug those four inequalities in the above inequality for $u_{j}$. After multiplying by $2^{j s}$ and summing up over $j$, we get

$$
\begin{aligned}
& \|u\|_{L_{t}^{\infty}\left(\dot{B}_{p, 1}^{s}\right)}+c_{p} \alpha\|u\|_{L_{t}^{1}\left(\dot{B}_{p, 1}^{s+2}\right)} \leq\left\|u_{0}\right\|_{\dot{B}_{p, 1}^{s}}+\|f\|_{L_{t}^{1}\left(\dot{B}_{p, 1}^{s}\right)} \\
& +C\left(\left\|\left(\operatorname{Id}-\dot{S}_{m}\right)(a b)\right\|_{L_{t}^{\infty}\left(\dot{B}_{p, 1}^{n / p}\right)}+\left\|\left(\operatorname{Id}-\dot{S}_{m}\right)(b \nabla a)\right\|_{L_{t}^{\infty}\left(\dot{B}_{p, 1}^{n / p-1}\right)}\right)\|u\|_{L_{t}^{1}\left(\dot{B}_{p, 1}^{s+2}\right)} \\
& +C \int_{0}^{t}\left\|\dot{S}_{m}(a \nabla b, b \nabla a)\right\|_{\dot{B}_{p, 1}^{n / p}}\|\nabla u\|_{\dot{B}_{p, 1}^{s}} d \tau .
\end{aligned}
$$

It is clear that, under Condition (3.10), the second line may be absorbed by the left-hand side. Hence the desired inequality follows from the interpolation inequality (3.8), exactly as in the smooth case.

We now look at the following Lamé system with nonconstant coefficients:

$$
\partial_{t} u-2 a \operatorname{div}(\mu D(u))-b \nabla(\lambda \operatorname{div} u)=f .
$$

Note that $u$ and $f$ are valued in $\mathbb{R}^{n}$. We assume throughout that the following uniform ellipticity condition is satisfied:

$$
\alpha:=\min \left(\inf _{(t, x) \in[0, T] \times \mathbb{R}^{n}}(a \mu)(t, x), \inf _{(t, x) \in[0, T] \times \mathbb{R}^{n}}(2 a \mu+b \lambda)(t, x)\right)>0 .
$$

Let us first study the "smooth case": 
Proposition 4. Assume that $a, b, \lambda$ and $\mu$ are bounded functions satisfying (3.14) and such that $a \nabla \mu, b \nabla \lambda, \mu \nabla a$ and $\lambda \nabla b$ are in $L^{2}\left(0, T ; \dot{B}_{p, 1}^{n / p}\right)$ for some $1<p<\infty$. There exists a constant $C$ such that the solutions to (3.13) satisfy for all $t \in[0, T]$,

$$
\begin{aligned}
\|u\|_{L_{t}^{\infty}\left(\dot{B}_{p, 1}^{s}\right)}+\alpha\|u\|_{L_{t}^{1}\left(\dot{B}_{p, 1}^{s+2}\right)} & \\
& \leq C\left(\left\|u_{0}\right\|_{\dot{B}_{p, 1}^{s}}+\|f\|_{L_{t}^{1}\left(\dot{B}_{p, 1}^{s}\right)}\right) \exp \left(\frac{C}{\alpha} \int_{0}^{t}\|(\mu \nabla a, a \nabla \mu, \lambda \nabla b, b \nabla \lambda)\|_{\dot{B}_{p, 1}^{n / p}}^{2} d \tau\right)
\end{aligned}
$$

whenever $-\min \left(n / p, n / p^{\prime}\right)<s \leq n / p$.

Proof. We introduce the following functions:

$$
d:=|D|^{-1} \operatorname{div} u \quad \text { and } \quad \Omega:=|D|^{-1} \operatorname{curl} u \text { with }(\operatorname{curl} u)_{i j}:=\partial_{i} u^{j}-\partial_{j} u^{i} .
$$

Owing to the use of homogeneous Besov space, and because the Fourier multipliers $A(D):=$ $|D|^{-1}$ div and $B(D):=|D|^{-1}$ curl are of degree 0 , it is equivalent to estimate $u$ or $(d, \Omega)$ in $L_{T}^{\infty}\left(\dot{B}_{p, 1}^{s}\right) \cap L_{T}^{1}\left(\dot{B}_{p, 1}^{s+2}\right)$. So the basic idea is to show that $d$ and $\Omega$ satisfy heat equations similar to (3.3). More precisely, applying $A(D)$ to (3.13) yields

$$
\begin{aligned}
\partial_{t} d-(2 a \mu+b \lambda) \Delta d=A(D)(f+2 a \nabla \mu \cdot D(u) & +b \nabla \lambda \operatorname{div} u) \\
& +[A(D), a \mu] \Delta u+[A(D), a \mu+b \lambda] \nabla \operatorname{div} u
\end{aligned}
$$

Given Condition (3.14), we see that arguing exactly as for proving (3.7) and because $A(D)$ maps $\dot{B}_{p, 1}^{s}$ in itself,

$$
\begin{aligned}
& \|d\|_{L_{t}^{\infty}\left(\dot{B}_{p, 1}^{s}\right)}+\kappa \alpha\|d\|_{L_{t}^{1}\left(\dot{B}_{p, 1}^{s+2}\right)} \leq\left\|d_{0}\right\|_{\dot{B}_{p, 1}^{s}}+\|A(D) f\|_{L_{t}^{1}\left(\dot{B}_{p, 1}^{s}\right)}+C \int_{0}^{t}\|2 a \nabla \mu \cdot D(u)+b \nabla \lambda \operatorname{div} u\|_{\dot{B}_{p, 1}^{s}} d \tau \\
& +C \int_{0}^{t}\|[A(D), a \mu] \Delta u+[A(D), a \mu+b \lambda] \nabla \operatorname{div} u\|_{\dot{B}_{p, 1}^{s}} d \tau+C \int_{0}^{t}\|\nabla(2 a \mu+b \lambda)\|_{\dot{B}_{p, 1}^{n / p}}\|\nabla u\|_{\dot{B}_{p, 1}^{s}} d \tau .
\end{aligned}
$$

Note that applying Lemma 6 with $\sigma=s-1, \nu=0$ and Lemma 4 with $\sigma=s$ and $\nu=0$ yields

$$
\begin{aligned}
& \|[A(D), a \mu] \Delta u\|_{\dot{B}_{p, 1}^{s}} \leq C\|\nabla(a \mu)\|_{\dot{B}_{p, 1}^{n / p}}\|\Delta u\|_{\dot{B}_{p, 1}^{s-1}}, \\
& \|a \nabla \mu \cdot D(u)\|_{\dot{B}_{p, 1}^{s}} \leq C\|a \nabla \mu\|_{\dot{B}_{p, 1}^{n / p}}\|\nabla u\|_{\dot{B}_{p, 1}^{s}},
\end{aligned}
$$

and analogous estimates for $[A(D), a \mu+b \lambda] \nabla \operatorname{div} u$ and $b \nabla \lambda \operatorname{div} u$.

Similarly, the vorticity part $\Omega$ of $u$ satisfies

$$
\partial_{t} \Omega-a \mu \Delta \Omega=B(D)(f+2 a \nabla \mu \cdot D(u)+b \nabla \lambda \operatorname{div} u)+[B(D), a \mu] \Delta u+[B(D), a \mu+b \lambda] \nabla \operatorname{div} u .
$$

So arguing exactly as for bounding $d$, and resorting to the interpolation inequality (3.8) and to Gronwall lemma, we easily get the desired inequality. It is just a matter of following the proof for the case of the heat equation.

Let us finally focus on the "rough case" where the coefficients of $(3.13)$ are only in $L_{T}^{\infty}\left(\dot{B}_{p, 1}^{n / p}\right)$.

Proposition 5. Let $a, b, \lambda$ and $\mu$ be bounded functions satisfying (3.14). Assume that $a \nabla \mu$, $b \nabla \lambda, \mu \nabla a$ and $\lambda \nabla b$ are in $L^{\infty}\left(0, T ; \dot{B}_{p, 1}^{n / p-1}\right)$ for some $1<p<\infty$. There exist two constants $\eta$ and $\kappa$ such that if for some $m \in \mathbb{Z}$ we have

$$
\begin{gathered}
\min \left(\inf _{(t, x) \in[0, T] \times \mathbb{R}^{n}} \dot{S}_{m}(2 a \mu+b \lambda)(t, x), \inf _{(t, x) \in[0, T] \times \mathbb{R}^{n}} \dot{S}_{m}(a \mu)(t, x)\right) \geq \frac{\alpha}{2}, \\
\left\|\left(\mathrm{Id}-\dot{S}_{m}\right)(\mu \nabla a, a \nabla \mu, \lambda \nabla b, b \nabla \lambda)\right\|_{L_{T}^{\infty}\left(\dot{B}_{p, 1}^{n / p-1}\right)} \leq \eta \alpha
\end{gathered}
$$


then the solutions to (3.13) satisfy for all $t \in[0, T]$,

$$
\begin{aligned}
\|u\|_{L_{t}^{\infty}\left(\dot{B}_{p, 1}^{s}\right)}+ & \alpha\|u\|_{L_{t}^{1}\left(\dot{B}_{p, 1}^{s+2}\right)} \\
& \leq C\left(\left\|u_{0}\right\|_{\dot{B}_{p, 1}^{s}}+\|f\|_{L_{t}^{1}\left(\dot{B}_{p, 1}^{s}\right)}\right) \exp \left(\frac{C}{\alpha} \int_{0}^{t}\left\|\dot{S}_{m}(\mu \nabla a, a \nabla \mu, \lambda \nabla b, b \nabla \lambda)\right\|_{\dot{B}_{p, 1}^{n / p}}^{2} d \tau\right)
\end{aligned}
$$

whenever $-\min \left(n / p, n / p^{\prime}\right)<s \leq n / p-1$.

Proof. As for the heat equation, we split the coefficients of the system into a smooth (but large) low frequency part and a rough (but small) high frequency part. It turns out to be more convenient to work directly on the equations for $d$ and $\Omega$. More precisely, as regards $d$, we write (starting from (3.15) and denoting $c:=2 a \mu+b \lambda$ ) that

$\partial_{t} d-\operatorname{div}(c \nabla d)=-\nabla c \cdot \nabla d+A(D)(f+2 a \nabla \mu \cdot D(u)+b \nabla \lambda \operatorname{div} u)$

$$
+[A(D), a \mu] \Delta u+[A(D), a \mu+b \lambda] \nabla \operatorname{div} u,
$$

whence, denoting $d_{j}:=\dot{\Delta}_{j} d$,

$$
\begin{aligned}
& \partial_{t} d_{j}-\operatorname{div}\left(\dot{S}_{m} c \nabla d_{j}\right)=\operatorname{div}\left(\left[\dot{\Delta}_{j}, \dot{S}_{m} c\right] \nabla d\right) \\
& +\dot{\Delta}_{j}\left(\operatorname{div}\left(\left(\mathrm{Id}-\dot{S}_{m}\right) c \nabla d\right)-\dot{S}_{m} \nabla c \cdot \nabla d-\left(\mathrm{Id}-\dot{S}_{m}\right) \nabla c \cdot \nabla d\right) \\
& \quad+\dot{\Delta}_{j} A(D)\left(f+2 \dot{S}_{m}(a \nabla \mu) \cdot D(u)+2\left(\mathrm{Id}-\dot{S}_{m}\right)(a \nabla \mu) \cdot D(u)\right. \\
& \left.+\dot{S}_{m}(b \nabla \lambda) \operatorname{div} u+\left(\operatorname{Id}-\dot{S}_{m}\right)(b \nabla \lambda) \operatorname{div} u\right)+\dot{\Delta}_{j}\left(\left[A(D), \dot{S}_{m}(a \mu)\right] \Delta u\right. \\
& \left.+\left[A(D), \dot{S}_{m}(a \mu+b \lambda)\right] \nabla \operatorname{div} u+\left[A(D),\left(\operatorname{Id}-\dot{S}_{m}\right)(a \mu)\right] \Delta u+\left[A(D),\left(\operatorname{Id}-\dot{S}_{m}\right)(a \mu+b \lambda)\right] \nabla \operatorname{div} u\right) .
\end{aligned}
$$

Under Condition (3.11), Lemmas 4, 5 and 6 imply that

$$
\begin{array}{ll}
\left\|\operatorname{div}\left(\left[\dot{\Delta}_{j}, \dot{S}_{m} c\right] \nabla d\right)\right\|_{L^{p}} & \lesssim c_{j} 2^{-j s}\left\|\dot{S}_{m} \nabla c\right\|_{\dot{B}_{p, 1}^{n / p}}\|\nabla d\|_{\dot{B}_{p, 1}^{s},}, \\
\left\|\dot{\Delta}_{j} \operatorname{div}\left(\left(\operatorname{Id}-\dot{S}_{m}\right) c \nabla d\right)\right\|_{L^{p}} & \lesssim c_{j} 2^{-j s}\left\|\left(\operatorname{Id}-\dot{S}_{m}\right) c\right\|_{\dot{B}_{p, 1}^{n / p}}\|\nabla d\|_{\dot{B}_{p, 1}^{s+1}}, \\
\left\|\dot{\Delta}_{j}\left(\dot{S}_{m} \nabla c \cdot \nabla d\right)\right\|_{L^{p}} & \lesssim c_{j} 2^{-j s}\left\|\dot{S}_{m} \nabla c\right\|_{\dot{B}_{p, 1}^{n / p}}\|\nabla d\|_{\dot{B}_{p, 1}^{s}}, \\
\| \dot{\Delta}_{j}\left(\left(\mathrm{Id}-\dot{S}_{m}\right) \nabla c \cdot \nabla d\right) & \lesssim c_{j} 2^{-j s}\left\|\left(\operatorname{Id}-\dot{S}_{m}\right) \nabla c\right\|_{\dot{B}_{p, 1}^{n / p-1}}\|\nabla d\|_{\dot{B}_{p, 1}^{s+1},} \\
\left\|\dot{\Delta}_{j}\left[A(D), \dot{S}_{m}(a \mu)\right] \Delta u\right\|_{L^{p}} & \lesssim c_{j} 2^{-j s}\left\|\nabla \dot{S}_{m}(a \mu)\right\|_{\dot{B}_{p, 1}^{n / p}}\|\Delta u\|_{\dot{B}_{p, 1}^{s-1}}, \\
\left\|\dot{\Delta}_{j}\left[A(D),\left(\operatorname{Id}-\dot{S}_{m}\right)(a \mu)\right] \Delta u\right\|_{L^{p}} & \lesssim c_{j} 2^{-j s}\left\|\nabla\left(\operatorname{Id}-\dot{S}_{m}\right)(a \mu)\right\|_{\dot{B}_{p, 1}^{n / p-1}}\|\Delta u\|_{\dot{B}_{p, 1}^{s}},
\end{array}
$$

and similar estimates for

$$
\begin{aligned}
\dot{\Delta}_{j} A(D)\left(\dot{S}_{m}(a \nabla \mu) \cdot D(u)\right), & \dot{\Delta}_{j} A(D)\left(\left(\mathrm{Id}-\dot{S}_{m}\right)(a \nabla \mu) \cdot D(u)\right), \\
\dot{\Delta}_{j} A(D)\left(\dot{S}_{m}(b \nabla \lambda) \operatorname{div} u\right), & \dot{\Delta}_{j} A(D)\left(\left(\mathrm{Id}-\dot{S}_{m}\right)(b \nabla \lambda) \operatorname{div} u\right), \\
\dot{\Delta}_{j}\left[A(D), \dot{S}_{m}(a \mu+b \lambda)\right] \nabla \operatorname{div} u, & \dot{\Delta}_{j}\left[A(D),\left(\operatorname{Id}-\dot{S}_{m}\right)(a \mu+b \lambda)\right] \nabla \operatorname{div} u .
\end{aligned}
$$

The curl part $\Omega$ of the velocity may be treated in the same way. Therefore we get

$$
\begin{aligned}
& \|u\|_{L_{t}^{\infty}\left(\dot{B}_{p, 1}^{s}\right)}+\alpha\|u\|_{L_{t}^{1}\left(\dot{B}_{p, 1}^{s+2}\right)} \lesssim\left\|u_{0}\right\|_{\dot{B}_{p, 1}^{s}}+\|f\|_{L_{t}^{1}\left(\dot{B}_{p, 1}^{s}\right)} \\
& \quad+\int_{0}^{t}\left\|\dot{S}_{m}(a \nabla \mu, \mu \nabla a, b \nabla \lambda, \lambda \nabla b)\right\|_{\dot{B}_{p, 1}^{n / p}}\|u\|_{\dot{B}_{p, 1}^{s+1}} d \tau \\
& \quad+\int_{0}^{t}\left\|\left(\mathrm{Id}-\dot{S}_{m}\right)(a \nabla \mu, \mu \nabla a, b \nabla \lambda, \lambda \nabla b)\right\|_{\dot{B}_{p, 1}^{n / p-1}}\|u\|_{\dot{B}_{p, 1}^{s+2}} d \tau .
\end{aligned}
$$

Obviously the last term may be absorbed by the left-hand side if $\eta$ is small enough in (3.17) and the last-but-one term may be handled by interpolation according to (3.8). So applying Gronwall lemma yields the desired inequality. 
For the sake of completeness, we still have to justify the existence of a solution to (3.13). More precisely, we want to establish the following result:

Proposition 6. Let $p$ be in $(1,+\infty)$. Let $a, b, \lambda$ and $\mu$ be bounded functions satisfying (3.14). Assume in addition that there exist some constants $\bar{a}, \bar{b}, \bar{\lambda}$ and $\bar{\mu}$ such that

$$
2 \bar{a} \bar{\mu}+\bar{b} \bar{\lambda}>0 \text { and } \bar{a} \bar{\mu}>0,
$$

and such that $a-\bar{a}, b-\bar{b}, \mu-\bar{\mu}$ and $\lambda-\bar{\lambda}$ are in $\mathcal{C}\left([0, T] ; \dot{B}_{p, 1}^{n / p}\right)$. Finally, suppose that

$$
\lim _{m \rightarrow+\infty}\left\|\left(\mathrm{Id}-\dot{S}_{m}\right)(a-\bar{a}, b-\bar{b}, \lambda-\bar{\lambda}, \mu-\bar{\mu})\right\|_{L_{T}^{\infty}\left(\dot{B}_{p, 1}^{n / p}\right)}=0 .
$$

Then for any data $u_{0} \in \dot{B}_{p, 1}^{s}$ and $f \in L^{1}\left(0, T ; \dot{B}_{p, 1}^{s}\right)$ with s satisfying (3.11), System (3.13) admits a unique solution $u \in \mathcal{C}\left([0, T] ; \dot{B}_{p, 1}^{s}\right) \cap L^{1}\left(0, T ; \dot{B}_{2,1}^{s+2}\right)$. Besides, the estimates of Proposition 5 are fulfilled for all large enough $m \in \mathbb{Z}$.

Proof. The proof is based on the continuity method as explained in e.g. [19] (and used in [11] in a similar context as ours). For $\theta \in[0,1]$, we introduce the following second order operator $\mathcal{P}_{\theta}$ acting on vector-fields $u$ as follows:

$$
\mathcal{P}_{\theta} u:=-2 a_{\theta} \operatorname{div}\left(\mu_{\theta} D(u)\right)-b_{\theta} \nabla\left(\lambda_{\theta} \operatorname{div} u\right)
$$

where $a_{\theta}:=(1-\theta) \bar{a}+\theta a, b_{\theta}:=(1-\theta) \bar{b}+\theta b$, and so on. We claim that one may find some $m \in \mathbb{Z}$ independent of $\theta$ such that for all $\theta \in[0,1]$, the conditions (3.16) and (3.17) are satisfied by $a_{\theta}, b_{\theta}, \mu_{\theta}$ and $\lambda_{\theta}$. Indeed, we notice that

$$
a_{\theta}-\bar{a}=\theta(a-\bar{a}) .
$$

Hence, for all $\theta \in[0,1]$,

$$
\left\|\left(\operatorname{Id}-\dot{S}_{m}\right)\left(a_{\theta}-\bar{a}\right)\right\|_{L_{T}^{\infty}\left(\dot{B}_{p, 1}^{n / p}\right)} \leq\left\|\left(\operatorname{Id}-\dot{S}_{m}\right)(a-\bar{a})\right\|_{L_{T}^{\infty}\left(\dot{B}_{p, 1}^{n / p}\right)}
$$

and similar properties hold for $b_{\theta}, \lambda_{\theta}$ and $\mu_{\theta}$. In particular, owing to the continuous embedding of $\dot{B}_{p, 1}^{n / p}$ in the set of continuous bounded functions, and to (3.19), we deduce that there exists some $m \in \mathbb{Z}$ so that the ellipticity condition (3.16) is satisfied by operator $\mathcal{P}_{\theta}$ for all $\theta \in[0,1]$.

Likewise, we have for instance

$$
\mu_{\theta} \nabla a_{\theta}=\theta(1-\theta) \bar{\mu} \nabla a+\theta^{2} \mu \nabla a
$$

and similar relations for the other coefficients. Hence one may find some large enough $m$ so that (3.17) is satisfied for all $\theta \in[0,1]$. In addition, the above relation shows that

$$
\left\|\dot{S}_{m}\left(\mu_{\theta} \nabla a_{\theta}\right)\right\|_{\dot{B}_{p, 1}^{n / p}} \leq \bar{\mu}\left\|\dot{S}_{m} \nabla a\right\|_{\dot{B}_{p, 1}^{n / p}}+\left\|\dot{S}_{m}(\mu \nabla a)\right\|_{\dot{B}_{p, 1}^{n / p}}
$$

Hence all the terms appearing in the exponential term of the estimate in Proposition 5 may be bounded by a constant depending only on $m$ and on the coefficients $a, b, \lambda$ and $\mu$. As a conclusion, one may thus find some constant $C$ independent of $\theta$ such that any solution $w$ of

$$
\partial_{t} w-\mathcal{P}_{\theta} w=g,\left.\quad w\right|_{t=0}=w_{0}
$$

satisfies

$$
\|w\|_{L_{T}^{\infty}\left(\dot{B}_{p, 1}^{s}\right)}+\alpha\|w\|_{L_{T}^{1}\left(\dot{B}_{p, 1}^{s+2}\right)} \leq C\left(\left\|w_{0}\right\|_{\dot{B}_{p, 1}^{s}}+\|g\|_{L_{T}^{1}\left(\dot{B}_{p, 1}^{s}\right)}\right)
$$

After this preliminary work, one may start with the proof of existence (uniqueness follows from the estimates of Proposition 5). Let $\mathcal{E}$ be the set of those $\theta$ in $[0,1]$ such that for every data $u_{0}$ and $f$ (as in the statement of the theorem), System

$$
\partial_{t} u-\mathcal{P}_{\theta} u=f,\left.\quad u\right|_{t=0}=u_{0}
$$

has a solution $u$ in the set $F_{p}^{s}(T):=\mathcal{C}\left([0, T] ; \dot{B}_{p, 1}^{s}\right) \cap L^{1}\left(0, T ; \dot{B}_{p, 1}^{s+2}\right)$. 
Note that according to Proposition 1 , the set $\mathcal{E}$ contains 0 hence is nonempty. So it suffices to find a fixed $\varepsilon>0$ such that for all $\theta_{0} \in \mathcal{E}$, we have

$$
\left[\theta_{0}-\varepsilon, \theta_{0}+\varepsilon\right] \cap[0,1] \subset \mathcal{E} .
$$

So let us fix some $\theta_{0} \in \mathcal{E}, u_{0} \in \dot{B}_{p, 1}^{s}, f \in L^{1}\left(0, T ; \dot{B}_{p, 1}^{s}\right)$ and $v \in F_{p}^{s}(T)$ and consider the solution $u$ to the system

$$
\partial_{t} u-\mathcal{P}_{\theta_{0}} u=f+\left(\mathcal{P}_{\theta}-\mathcal{P}_{\theta_{0}}\right) v
$$

with $\theta \in[0,1]$ such that $\left|\theta-\theta_{0}\right| \leq \varepsilon$. Given that $\theta_{0}$ is in $\mathcal{E}$, the existence of $u$ in $F_{p}^{s}(T)$ is granted if $\left(\mathcal{P}_{\theta}-\mathcal{P}_{\theta_{0}}\right) v \in L^{1}\left(0, T ; \dot{B}_{p, 1}^{s}\right)$. So let us first check this: we have

$$
\begin{aligned}
\left(\mathcal{P}_{\theta}-\mathcal{P}_{\theta_{0}}\right) v=\left(\theta-\theta_{0}\right)\left(2 a_{\theta_{0}} \operatorname{div}((\bar{\mu}-\mu) D(v))+\right. & 2(\bar{a}-a) \operatorname{div}\left(\mu_{\theta} D(v)\right) \\
& \left.+b_{\theta_{0}} \nabla((\bar{\lambda}-\lambda) \operatorname{div} v)+(\bar{b}-b) \nabla\left(\lambda_{\theta} \operatorname{div} v\right)\right) .
\end{aligned}
$$

Under Condition (3.11), one may thus conclude thanks to product estimates in Besov spaces (see Lemma 4) that $\left(\mathcal{P}_{\theta}-\mathcal{P}_{\theta_{0}}\right) v \in L^{1}\left(0, T ; \dot{B}_{p, 1}^{s}\right)$. Furthermore

$$
\begin{aligned}
\left\|\left(\mathcal{P}_{\theta}-\mathcal{P}_{\theta_{0}}\right) v\right\|_{\dot{B}_{p, 1}^{s}} \leq C \varepsilon\left(\left(\bar{a}+\left\|a_{\theta_{0}}-\bar{a}\right\|_{\dot{B}_{p, 1}^{n / p}}\right)\|\mu-\bar{\mu}\|_{\dot{B}_{p, 1}^{n / p}}+\left(\bar{\mu}+\left\|\mu_{\theta}-\bar{\mu}\right\|_{\dot{B}_{p, 1}^{n / p}}\right)\|a-\bar{a}\|_{\dot{B}_{p, 1}^{n / p}}\right. \\
\left.+\left(\bar{b}+\left\|b_{\theta_{0}}-\bar{b}\right\|_{\dot{B}_{p, 1}^{n / p}}\right)\|\lambda-\bar{\lambda}\|_{\dot{B}_{p, 1}^{n / p}}+\left(\bar{\lambda}+\left\|\lambda_{\theta}-\bar{\lambda}\right\|_{\dot{B}_{p, 1}^{n / p}}\right)\|a-\bar{a}\|_{\dot{B}_{p, 1}^{n / p}}\right)\|D v\|_{\dot{B}_{p, 1}^{s+1}} .
\end{aligned}
$$

The coefficients may be bounded in terms of the initial coefficients $a, b, \lambda$ and $\mu$. Hence, applying (3.20) we get for some constant independent of $\theta_{0}$ and of $\theta$,

$$
\|u\|_{L_{T}^{\infty}\left(\dot{B}_{p, 1}^{s}\right)}+\alpha\|u\|_{L_{T}^{1}\left(\dot{B}_{p, 1}^{s+2}\right)} \leq C\left(\varepsilon\|v\|_{L_{T}^{1}\left(\dot{B}_{p, 1}^{s+2}\right)}+\left\|w_{0}\right\|_{\dot{B}_{p, 1}^{s}}+\|f\|_{L_{T}^{1}\left(\dot{B}_{p, 1}^{s}\right)}\right) .
$$

Taking $\varepsilon$ small enough, it becomes clear that the linear map $\Psi_{\theta}: v \mapsto u$ is contractive on the Banach space $F_{p}^{s}(T)$. Hence it has a (unique) fixed point $u \in F_{p}^{s}(T)$. In other words, $u$ satisfies (3.21).

Given that $\mathcal{E}$ is nonempty and that $\varepsilon$ is independent of $\theta_{0}$, one may now conclude that 1 is in $\mathcal{E}$. Therefore, there exists a solution $u \in F_{p}^{s}(T)$ to $(3.13)$.

Remark 1. Under the assumptions of the above proposition, the constructed solution u satisfies $\partial_{t} u \in L^{1}\left(0, T ; \dot{B}_{p, 1}^{s}\right)$. Indeed, it suffices to notice that

$$
\partial_{t} u=f+(\bar{a}+(a-\bar{a})) \operatorname{div}(\bar{\mu}+(\mu-\bar{\mu}) D(u))+(\bar{b}+(b-\bar{b})) \nabla(\bar{\lambda}+(\lambda-\bar{\lambda}) \operatorname{div} u),
$$

and to use Lemma 4 together with the facts that $\nabla u$ is in $L^{1}\left(0, T ; \dot{B}_{p, 1}^{s+1}\right)$. Moreover we have

$$
\left\|\partial_{t} u\right\|_{L_{T}^{1}\left(\dot{B}_{p, 1}^{s}\right)} \leq C\left(\left\|u_{0}\right\|_{\dot{B}_{p, 1}^{s}}+\|f\|_{L_{t}^{1}\left(\dot{B}_{p, 1}^{s}\right)}\right) \exp \left(\frac{C}{\alpha} \int_{0}^{t}\left\|\dot{S}_{m}(\mu \nabla a, a \nabla \mu, \lambda \nabla b, b \nabla \lambda)\right\|_{\dot{B}_{p, 1}^{n / p}}^{2} d \tau\right)
$$

where $C$ may depend also on the norm of $a-\bar{a}, b-\bar{b}, \lambda-\bar{\lambda}$ and $\mu-\bar{\mu}$ in $L^{\infty}\left(0, T ; \dot{B}_{p, 1}^{n / p}\right)$.

3.2. Proof of Theorem 1. As we want to consider (possibly) large velocities, we introduce, as in the almost homogeneous case the free solution to the Lamé system corresponding to $\rho \equiv 1$, that is the vector-field $u_{L}$ in $E_{p}(T)$, given by Proposition 1, satisfying ${ }^{2}$

$$
L_{1} u_{L}=0,\left.\quad u\right|_{t=0}=u_{0} .
$$

We claim that the Banach fixed point theorem applies to the map $\Phi$ defined in (3.2) in some closed ball $\bar{B}_{E_{p}(T)}\left(u_{L}, R\right)$ with suitably small $T$ and $R$. Denoting $\widetilde{u}:=u-u_{L}$, we see that $\widetilde{u}$ has to satisfy

$$
\left\{\begin{array}{l}
L_{\rho_{0}} \widetilde{u}=\rho_{0}^{-1} \operatorname{div}\left(I_{1}(v, v)+I_{2}(v, v)+I_{3}(v, v)+I_{4}(v)\right)+\left(L_{1}-L_{\rho_{0}}\right) u_{L}, \\
\left.\widetilde{u}\right|_{t=0}=0 .
\end{array}\right.
$$

\footnotetext{
${ }^{2}$ See (3.1) for the definition of operator $L_{1}$.
} 
If the right-hand side is in $L^{1}\left(0, T ; \dot{B}_{p, 1}^{n / p-1}\right)$ and if there exists some $m \in \mathbb{Z}$ so that (3.16) and (3.17) are fulfilled then Proposition 6 and Remark 1 ensure the existence of $\widetilde{u}$ in $E_{p}(T)$. Now, the existence of $m$ so that

$$
\begin{gathered}
\min \left(\inf _{x} \dot{S}_{m}\left(2 \frac{\mu\left(\rho_{0}\right)}{\rho_{0}}+\frac{\lambda\left(\rho_{0}\right)}{\rho_{0}}\right), \inf _{x} \dot{S}_{m}\left(\frac{\mu\left(\rho_{0}\right)}{\rho_{0}}\right)\right)>\frac{\alpha}{2} \\
\text { and }\left\|\left(\operatorname{Id}-\dot{S}_{m}\right)\left(\frac{\mu\left(\rho_{0}\right)}{\rho_{0}^{2}} \nabla \rho_{0}, \frac{\mu^{\prime}\left(\rho_{0}\right)}{\rho_{0}} \nabla \rho_{0}, \frac{\lambda\left(\rho_{0}\right)}{\rho_{0}^{2}} \nabla \rho_{0}, \frac{\lambda^{\prime}\left(\rho_{0}\right)}{\rho_{0}} \nabla \rho_{0}\right)\right\|_{\dot{B}_{p, 1}^{n / p-1}} \leq \eta \alpha .
\end{gathered}
$$

is ensured by the fact that all the coefficients (minus some constant) belong to the space $\dot{B}_{p, 1}^{n / p}$ which is defined in terms of a convergent series and embeds continuously in the set of bounded continuous functions. The study of the right-hand side of (3.23) will be carried out below.

First step: Stability of $\bar{B}_{E_{p}(T)}\left(u_{L}, R\right)$ for small enough $R$ and $T$. Proposition 5 and the definition of the multiplier space $\mathcal{M}\left(\dot{B}_{p, 1}^{n / p-1}\right)$ ensure that

$$
\begin{aligned}
& \text { (3.24) }\|\widetilde{u}\|_{E_{p}(T)} \leq C e^{C_{\rho_{0}, m} T}\left(\left\|\left(L_{1}-L_{\rho_{0}}\right) u_{L}\right\|_{L_{T}^{1}\left(\dot{B}_{p, 1}^{n / p-1}\right)}\right. \\
& \left.+\left\|\rho_{0}^{-1}\right\|_{\mathcal{M}\left(\dot{B}_{p, 1}^{n / p-1}\right)}\left(\left\|I_{1}(v, v)\right\|_{L_{T}^{1}\left(\dot{B}_{p, 1}^{n / p}\right)}+\left\|I_{2}(v, v)\right\|_{L_{T}^{1}\left(\dot{B}_{p, 1}^{n / p}\right)}+\left\|I_{3}(v, v)\right\|_{L_{T}^{1}\left(\dot{B}_{p, 1}^{n / p}\right)}+\left\|I_{4}(v)\right\|_{L_{T}^{1}\left(\dot{B}_{p, 1}^{n / p}\right)}\right)\right)
\end{aligned}
$$

for some constant $C_{\rho_{0}, m}$ depending only on $\rho_{0}$ and on $m$.

In what follows, we assume that $T$ and $R$ have been chosen so that (2.7) is satisfied by $v$. Using the decomposition

$$
\begin{aligned}
\left(L_{1}-L_{\rho_{0}}\right) u_{L}=\left(\rho_{0}^{-1}-1\right) \operatorname{div}\left(2 \mu\left(\rho_{0}\right)\right. & \left.D\left(u_{L}\right)+\lambda\left(\rho_{0}\right) \operatorname{div} u_{L} \mathrm{Id}\right) \\
& +\operatorname{div}\left(2\left(\mu\left(\rho_{0}\right)-\mu(1)\right) D(u)+\left(\lambda\left(\rho_{0}\right)-\lambda(1)\right) \operatorname{div} u \mathrm{Id}\right),
\end{aligned}
$$

and composition inequalities (1.5) and (1.6), we see that $\left(L_{1}-L_{\rho_{0}}\right) u_{L} \in L^{1}\left(0, T ; \dot{B}_{p, 1}^{n / p-1}\right)$ and

$$
\left\|\left(L_{1}-L_{\rho_{0}}\right) u_{L}\right\|_{L_{T}^{1}\left(\dot{B}_{p, 1}^{n / p-1}\right)} \lesssim\left\|a_{0}\right\|_{\dot{B}_{p, 1}^{n / p}}\left(1+\left\|a_{0}\right\|_{\dot{B}_{p, 1}^{n / p}}\right)\left\|D u_{L}\right\|_{L_{T}^{1}\left(\dot{B}_{p, 1}^{n / p}\right)} .
$$

Likewise, flow and composition estimates ensure that

$$
\left\|I_{i}(v, w)\right\|_{L_{T}^{1}\left(\dot{B}_{p, 1}^{n / p-1}\right)} \lesssim\left(1+\left\|a_{0}\right\|_{\left.\dot{B}_{p, 1}^{n / p}\right)}\|D v\|_{L_{T}^{1}\left(\dot{B}_{p, 1}^{n / p}\right)}\|D w\|_{L_{T}^{1}\left(\dot{B}_{p, 1}^{n / p}\right)} \text { for } i=1,2,3\right.
$$

and that

$$
\left\|I_{4}(v)\right\|_{L_{T}^{1}\left(\dot{B}_{p, 1}^{n / p}\right)} \lesssim T\left(1+\left\|a_{0}\right\|_{\dot{B}_{p, 1}^{n / p}}\right)\left(1+\|D v\|_{L_{T}^{1}\left(\dot{B}_{p, 1}^{n / p}\right)}\right) .
$$

So plugging the above inequalities in (3.24) and keeping in mind that $v$ satisfies (2.7), we get after decomposing $v$ into $\widetilde{v}+u_{L}$ :

$$
\begin{aligned}
\|\widetilde{u}\|_{E_{p}(T)} \leq C e^{C_{\rho_{0}, m} T}(1+ & \left.\left\|a_{0}\right\|_{\dot{B}_{p, 1}^{n / p}}\right)^{2}\left(\left(T+\left\|a_{0}\right\|_{\dot{B}_{p, 1}^{n / p}}\left\|D u_{L}\right\|_{L_{T}^{1}\left(\dot{B}_{p, 1}^{n / p}\right)}\right)\right. \\
& \left.+\left\|D u_{L}\right\|_{L_{T}^{1}\left(\dot{B}_{p, 1}^{n / p}\right)}^{2}+\left(\left\|D u_{L}\right\|_{L_{T}^{1}\left(\dot{B}_{p, 1}^{n / p}\right)}+\|D \widetilde{v}\|_{L_{T}^{1}\left(\dot{B}_{p, 1}^{n / p}\right)}\right)\|D \widetilde{v}\|_{L_{T}^{1}\left(\dot{B}_{p, 1}^{n / p}\right)}\right) .
\end{aligned}
$$

So, because $\widetilde{v} \in B_{E_{p}(T)}\left(u_{L}, R\right)$,

$$
\begin{aligned}
\|\widetilde{u}\|_{E_{p}(T)} \leq C e^{C_{\rho_{0}, m} T}\left(1+\left\|a_{0}\right\|_{\dot{B}_{p, 1}^{n / p}}\right)^{2}\left(\left(T+\left\|a_{0}\right\|_{\dot{B}_{p, 1}^{n / p}}\left\|D u_{L}\right\|_{L_{T}^{1}\left(\dot{B}_{p, 1}^{n / p}\right)}\right)\right. & \\
& \left.+\left(R+\left\|D u_{L}\right\|_{L_{T}^{1}\left(\dot{B}_{p, 1}^{n / p}\right)}\right)\left\|D u_{L}\right\|_{L_{T}^{1}\left(\dot{B}_{p, 1}^{n / p}\right)}+R^{2}\right) .
\end{aligned}
$$

Therefore, if we first choose $R$ so that for a small enough constant $\eta$,

$$
\left(1+\left\|a_{0}\right\|_{\dot{B}_{p, 1}^{n / p}}\right)^{2} R \leq \eta
$$


and then take $T$ so that

$$
\begin{gathered}
C_{\rho_{0}, m} T \leq \log 2, \quad T \leq R^{2}, \quad\left\|a_{0}\right\|_{\dot{B}_{p, 1}^{n / p}}\left\|D u_{L}\right\|_{L_{T}^{1}\left(\dot{B}_{p, 1}^{n / p}\right)} \leq R^{2}, \\
\left\|D u_{L}\right\|_{L_{T}^{1}\left(\dot{B}_{p, 1}^{n / p}\right)} \leq R,
\end{gathered}
$$

then we may conclude that $\Phi$ maps $\bar{B}_{E_{p}(T)}\left(u_{L}, R\right)$ into itself.

Second step: contraction estimates. Let us now establish that, under Condition (3.29), the map $\Phi$ is contractive. We consider two vector-fields $v^{1}$ and $v^{2}$ in $\bar{B}_{E_{p}(T)}\left(u_{L}, R\right)$, and set $u^{1}:=\Phi\left(v^{1}\right)$ and $u^{2}:=\Phi\left(v^{2}\right)$. Let $\delta u:=u^{2}-u^{1}$ and $\delta v:=v^{2}-v^{1}$. In order to prove that $\Phi$ is contractive, it is mainly a matter of applying Proposition 5 to

$$
\begin{aligned}
L_{\rho_{0}} \delta u=\rho_{0}^{-1} \operatorname{div}\left(\left(I_{1}\left(v^{2}, v^{2}\right)-I_{1}\left(v^{1}, v^{1}\right)\right)\right. & \\
& \left.+\left(I_{2}\left(v^{2}, v^{2}\right)-I_{2}\left(v^{1}, v^{1}\right)\right)+\left(I_{3}\left(v^{2}, v^{2}\right)-I_{3}\left(v^{1}, v^{1}\right)\right)+\left(I_{4}\left(v^{2}\right)-I_{4}\left(v^{1}\right)\right)\right) .
\end{aligned}
$$

So we have, given that $C_{\rho_{0}, m} T \leq \log 2$,

$$
\begin{aligned}
& \text { (3.30) }\|\delta u\|_{E_{p}(T)} \leq C\left(1+\left\|a_{0}\right\|_{\dot{B}_{p, 1}^{n / p}}\right)\left(\left\|I_{1}\left(v^{2}, v^{2}\right)-I_{1}\left(v^{1}, v^{1}\right)\right\|_{L_{T}^{1}\left(\dot{B}_{p, 1}^{n / p}\right)}\right. \\
& \left.+\left\|I_{2}\left(v^{2}, v^{2}\right)-I_{2}\left(v^{1}, v^{1}\right)\right\|_{L_{T}^{1}\left(\dot{B}_{p, 1}^{n / p}\right)}+\left\|I_{3}\left(v^{2}, v^{2}\right)-I_{3}\left(v^{1}, v^{1}\right)\right\|_{L_{T}^{1}\left(\dot{B}_{p, 1}^{n / p}\right)}+\left\|I_{4}\left(v^{2}\right)-I_{4}\left(v^{1}\right)\right\|_{L_{T}^{1}\left(\dot{B}_{p, 1}^{n / p}\right)}\right) .
\end{aligned}
$$

In order to deal with the first term of the right-hand side, we use the decomposition

$$
\begin{aligned}
I_{1}\left(v^{2}, v^{2}\right)-I_{1}\left(v^{1}, v^{1}\right) & =\lambda\left(J_{v^{2}}^{-1} \rho_{0}\right)\left({ }^{T} A_{v^{2}}: \nabla v^{2}\right)\left(\operatorname{adj}\left(D X_{v^{2}}\right)-\operatorname{adj}\left(D X_{v^{1}}\right)\right) \\
& +\left(\operatorname{adj}\left(D X_{v^{1}}\right)-\operatorname{Id}\right)\left(\lambda\left(J_{v^{2}}^{-1} \rho_{0}\right)-\lambda\left(J_{v^{1}}^{-1} \rho_{0}\right)\right)\left({ }^{T} A_{v^{2}}: \nabla v^{2}\right) \\
& +\left(\operatorname{adj}\left(D X_{v^{1}}\right)-\operatorname{Id}\right) \lambda\left(J_{v^{1}}^{-1} \rho_{0}\right)\left(\left({ }^{T} A_{v^{2}}-{ }^{T} A_{v^{1}}\right): \nabla v^{1}+{ }^{T} A_{v^{2}}: \nabla \delta v\right) \\
& + \text { terms pertaining to } \mu .
\end{aligned}
$$

Taking advantage of product laws in Besov spaces, of composition estimates (1.5) and (1.6), and of the flow estimates in the appendix, we deduce that for some constant $C_{\rho_{0}}$ depending only on $\rho_{0}$ :

$$
\left\|I_{1}\left(v^{2}, v^{2}\right)-I_{1}\left(v^{1}, v^{1}\right)\right\|_{L_{T}^{1}\left(\dot{B}_{p, 1}^{n / p}\right)} \leq C_{\rho_{0}}\left\|\left(D v^{1}, D v^{2}\right)\right\|_{L_{T}^{1}\left(\dot{B}_{p, 1}^{n / p}\right)}\|D \delta v\|_{L_{T}^{1}\left(\dot{B}_{p, 1}^{n / p}\right)} .
$$

Similar estimates may be proved for the next two terms of the right-hand side of (3.30). Concerning the last one, we use the decomposition

$$
I_{4}\left(v^{2}\right)-I_{4}\left(v^{1}\right)=\left(\operatorname{adj}\left(D X_{v^{1}}\right)-\operatorname{adj}\left(D X_{v^{2}}\right)\right) P\left(J_{v^{2}}^{-1} \rho_{0}\right)-\operatorname{adj}\left(D X_{v^{1}}\right)\left(P\left(J_{v^{2}}^{-1} \rho_{0}\right)-P\left(J_{v^{1}}^{-1} \rho_{0}\right)\right) .
$$

Hence

$$
\left\|I_{4}\left(v^{2}\right)-I_{4}\left(v^{1}\right)\right\|_{L_{T}^{1}\left(\dot{B}_{p, 1}^{n / p}\right)} \leq C\left(1+\left\|a_{0}\right\|_{\left.\dot{B}_{p, 1}^{n / p}\right) T\|D \delta v\|_{L_{T}^{1}\left(\dot{B}_{p, 1}^{n / p}\right)}} .\right.
$$

We end up with

$$
\|\delta u\|_{E_{p}(T)} \leq C\left(1+\left\|a_{0}\right\|_{\dot{B}_{p, 1}^{n / p}}\right)^{2}\left(T+\left\|\left(D v^{1}, D v^{2}\right)\right\|_{L_{T}^{1}\left(\dot{B}_{p, 1}^{n / p}\right)}\right)\|D \delta v\|_{L_{T}^{1}\left(\dot{B}_{p, 1}^{n / p}\right)}
$$

Given that $v^{1}$ and $v^{2}$ are in $\bar{B}_{E_{p}(T)}\left(u_{L}, R\right)$, our hypotheses over $T$ and $R$ (with smaller $\eta$ in (3.28) if need be) thus ensure that, say,

$$
\|\delta u\|_{E_{p}(T)} \leq \frac{1}{2}\|\delta v\|_{E_{p}(T)} .
$$

One can thus conclude that $\Phi$ admits a unique fixed point in $\bar{B}_{E_{p}(T)}\left(u_{L}, R\right)$. 
Third step: Regularity of the density. Granted with the above velocity field $u$ in $E_{p}(T)$, we set $\rho:=J_{u}^{-1} \rho_{0}$. By construction, the couple $(\rho, u)$ satisfies (1.2). Let us now prove that $a:=\rho-1$ is in $\mathcal{C}\left([0, T] ; \dot{B}_{p, 1}^{n / p}\right)$. We have

$$
a=\left(J_{u}^{-1}-1\right) a_{0}+a_{0} .
$$

Given (A.11) and using the fact that $D u \in L^{1}\left(0, T ; \dot{B}_{p, 1}^{n / p}\right)$, it is clear that $J_{u}^{-1}-1$ belongs to $\mathcal{C}\left([0, T] ; \dot{B}_{p, 1}^{n / p}\right)$. Hence $a$ belongs to $\mathcal{C}\left([0, T] ; \dot{B}_{p, 1}^{n / p}\right)$, too. Because $\dot{B}_{p, 1}^{n / p}$ is continuously embedded in $L^{\infty}$, Condition (1.7) is fulfilled on $[0, T]$ (taking $T$ smaller if needed).

Last step: Uniqueness and continuity of the flow map. We now consider two couples $\left(\rho_{0}^{1}, u_{0}^{1}\right)$ and $\left(\rho_{0}^{2}, u_{0}^{2}\right)$ of data fulfilling the assumptions of Theorem 1 and we denote by $\left(\rho^{1}, u^{1}\right)$ and $\left(\rho^{2}, u^{2}\right)$ two solutions in $E_{p}(T)$ corresponding to those data. Setting $\delta u:=u^{2}-u^{1}$, we see that

$$
\begin{aligned}
L_{\rho_{0}^{1}}(\delta u)=\left(L_{\rho_{0}^{1}}-L_{\rho_{0}^{2}}\right)\left(u^{2}\right)+\left(\rho_{0}^{1}\right)^{-1} \operatorname{div}\left(\sum_{j=1}^{3}\right. & \left(\left(I_{j}^{2}\left(u^{2}, u^{2}\right)-I_{j}^{2}\left(u^{1}, u^{1}\right)\right)+\left(I_{4}^{2}\left(u^{2}\right)-I_{4}^{2}\left(u^{1}\right)\right)\right) \\
& +\left(\rho_{0}^{1}\right)^{-1} \operatorname{div}\left(\sum_{j=1}^{3}\left(\left(I_{j}^{2}-I_{j}^{1}\right)\left(u^{1}, u^{1}\right)+\left(I_{4}^{2}-I_{4}^{1}\right)\left(u^{1}\right)\right),\right.
\end{aligned}
$$

where $I_{1}^{i}, I_{2}^{i}, I_{3}^{i}$ and $I_{4}^{i}$ correspond to the quantities that have been defined just above (3.2), with density $\rho_{0}^{i}$. Note that those terms may be bounded exactly as in the second step. So the only definitely new terms are $\left(L_{\rho_{0}^{1}}-L_{\rho_{0}^{2}}\right)\left(u^{2}\right)$ and the last line. As regards $\left(L_{\rho_{0}^{1}}-L_{\rho_{0}^{2}}\right)\left(u^{2}\right)$, it may be decomposed into

$$
\begin{aligned}
\left(L_{\rho_{0}^{1}}-L_{\rho_{0}^{2}}\right)\left(u^{2}\right) & =\left(\left(\rho_{0}^{1}\right)^{-1}-\left(\rho_{0}^{2}\right)^{-1}\right) \operatorname{div}\left(2 \mu\left(\rho_{0}^{1}\right) D\left(u^{2}\right)+\lambda\left(\rho_{0}^{1}\right) \operatorname{div} u^{2} \mathrm{Id}\right) \\
& -\left(\rho_{0}^{2}\right)^{-1} \operatorname{div}\left(2\left(\mu\left(\rho_{0}^{2}\right)-\mu\left(\rho_{0}^{1}\right)\right) D\left(u^{2}\right)+\left(\lambda\left(\rho_{0}^{2}\right)-\lambda\left(\rho_{0}^{1}\right)\right) \operatorname{div} u^{2} \mathrm{Id}\right) .
\end{aligned}
$$

Hence, combining composition, flow and product estimates, we get for $t \leq T$,

$$
\left\|\left(L_{\rho_{0}^{1}}-L_{\rho_{0}^{2}}\right)\left(u^{2}\right)\right\|_{L_{t}^{1}\left(\dot{B}_{p, 1}^{n / p-1}\right)} \leq C_{\rho_{0}^{1}, \rho_{0}^{2}}\left\|\delta \rho_{0}\right\|_{\dot{B}_{p, 1}^{n / p}}\left\|D u^{2}\right\|_{L_{t}^{1}\left(\dot{B}_{p, 1}^{n / p}\right)} .
$$

It is not difficult to show that the other "new" terms satisfy analogous estimates. Hence, applying Proposition 5 to the system that is satisfied by $\delta u$, we discover that for $t \leq T$,

$$
\begin{aligned}
\|\delta u\|_{E_{p}(t)} \leq C_{\rho_{0}^{1}, \rho_{0}^{2}}\left(\left(t+\left\|\left(D u^{1}, D u^{2}\right)\right\|_{L_{t}^{1}\left(\dot{B}_{p, 1}^{n / p}\right)}\right)\|D \delta u\|_{L_{t}^{1}\left(\dot{B}_{p, 1}^{n / p}\right)}\right. \\
\left.+\left\|\delta u_{0}\right\|_{\dot{B}_{p, 1}^{n / p}}+\left\|\delta \rho_{0}\right\|_{\dot{B}_{p, 1}^{n / p}}\left(t+\left\|\left(D u^{1}, D u^{2}\right)\right\|_{L_{t}^{1}\left(\dot{B}_{p, 1}^{n / p}\right)}\right)\right) .
\end{aligned}
$$

Let us emphasize that the constant $C_{\rho_{0}^{1}, \rho_{0}^{2}}$ depends only on $\rho_{0}^{2}$ through its norm, for the integer $m$ used in Proposition 5 corresponds to $\rho_{0}^{1}$ only. Hence if $\delta \rho_{0}$ is small enough then the above inequality recasts in

$$
\begin{aligned}
\|\delta u\|_{E_{p}(t)} \leq C_{\rho_{0}^{1}}\left(\left(t+\left\|D u^{1}\right\|_{L_{t}^{1}\left(\dot{B}_{p, 1}^{n / p}\right)}+\|\delta u\|_{E_{p}(t)}\right)\|\delta u\|_{E_{p}(t)}\right. & \\
& \left.+\left\|\delta u_{0}\right\|_{\dot{B}_{p, 1}^{n / p}}+\left\|\delta \rho_{0}\right\|_{\dot{B}_{p, 1}^{n / p}}\left(t+\left\|D u^{1}\right\|_{L_{t}^{1}\left(\dot{B}_{p, 1}^{n / p}\right)}\right)\right) .
\end{aligned}
$$

An obvious bootstrap argument thus shows that if $t, \delta u_{0}$ and $\delta \rho_{0}$ are small enough then

$$
\|\delta u\|_{E_{p}(t)} \leq 2 C_{\rho_{0}}\left(\left\|\delta u_{0}\right\|_{\dot{B}_{p, 1}^{n / p}}+\left\|\delta \rho_{0}\right\|_{\dot{B}_{p, 1}^{n / p}}\right) .
$$

As regards the density, we have

$$
\delta a=J_{u^{1}}^{-1} \delta a_{0}+\left(J_{u^{2}}^{-1}-J_{u^{1}}^{-1}\right) a_{0}^{2} .
$$

Hence for all $t \in[0, T]$,

$$
\|\delta a(t)\|_{\dot{B}_{p, 1}^{n / p}} \leq C\left(1+\left\|D u^{1}\right\|_{L_{t}^{1}\left(\dot{B}_{p, 1}^{n / p}\right)}\right)\left\|\delta a_{0}\right\|_{\dot{B}_{p, 1}^{n / p}}\|D \delta u\|_{L_{t}^{1}\left(\dot{B}_{p, 1}^{n / p}\right)} .
$$


So we eventually get uniqueness and continuity of the flow map on a small enough time interval. Then iterating the proof yields uniqueness on the initial time interval $[0, T]$. Note that it also yields Lipschitz continuity of the flow map for the velocity as for fixed data $\left(\rho_{0}^{1}, u_{0}^{1}\right)$, one may find some neighborhood and common time interval on which all the solutions constructed in the previous steps exist.

3.3. Proof of Theorem 2. For $u_{0} \in \dot{B}_{p, 1}^{n / p-1}$ and $\rho_{0} \in\left(1+\dot{B}_{p, 1}^{n / p}\right)$, the local existence for (0.1) may be proved directly (see $[4,8]$ ) but only under the assumption that $p \leq n$ in the case of nonconstant viscosity coefficients. Here we get the result (including uniqueness) from Theorem 1 , and under the sole assumption that $p<2 n$. This is a mere corollary of the following proposition which states the equivalence of the systems (0.1) and (1.2) in our functional setting.

Proposition 7. Assume that the couple $(\rho, u)$ with $(\rho-1) \in \mathcal{C}\left([0, T] ; \dot{B}_{p, 1}^{n / p}\right)$ and $u \in E_{p}(T)$ (with $1 \leq p<2 n$ ) is a solution to (0.1) such that

$$
\int_{0}^{T}\|\nabla u\|_{\dot{B}_{p, 1}^{n / p}} d t \leq c
$$

Let $X$ be the flow of $u$ defined in (1.1). Then the couple $(\bar{\rho}, \bar{u}):=(\rho \circ X, u \circ X)$ belongs to the same functional space as $(\rho, u)$, and satisfies (1.2).

Conversely, if $(\bar{\rho}-1, \bar{u})$ belongs to $\mathcal{C}\left([0, T] ; \dot{B}_{p, 1}^{n / p}\right) \times E_{p}(T)$ and $(\bar{\rho}, \bar{u})$ satisfies (1.2) and, for a small enough constant $c$,

$$
\int_{0}^{T}\|\nabla \bar{u}\|_{\dot{B}_{p, 1}^{n / p}} d t \leq c
$$

then the map $X$ defined in (1.3) is a $C^{1}$ (and in fact a locally $\dot{B}_{p, 1}^{n / p+1}$ ) diffeomorphism over $\mathbb{R}^{n}$ and the couple $(\rho, u):=\left(\bar{\rho} \circ X^{-1}, \bar{u} \circ X^{-1}\right)$ satisfies $(0.1)$ and has the same regularity as $(\bar{\rho}, \bar{u})$.

Proof. Let us first consider a solution $(\rho, u)$ to $(0.1)$ with the above properties. Then, the definition of $X$ implies that $D X-$ Id is in $\mathcal{C}\left([0, T] ; \dot{B}_{p, 1}^{n / p}\right)$. In addition, Proposition 8 ensures that $(\bar{\rho}, \bar{u}):=(\rho \circ X, u \circ X)$ belongs to the same functional space as $(\rho, u)$, and (A.9), (A.10), (A.11) below imply that $A-\mathrm{Id}, \operatorname{adj}(D X)-\mathrm{Id}$ and $J^{-1}-1$ are in $\mathcal{C}\left([0, T] ; \dot{B}_{p, 1}^{n / p}\right)$. Therefore the product laws for Besov spaces enable us to use the algebraic relations (A.5), (A.6), (A.7) and (A.8) whenever $p<2 n$. Therefore $(\bar{\rho}, \bar{u})$ fulfills $(1.2)$.

Conversely, if we are given some solution $(\bar{\rho}, \bar{u})$ in $\mathcal{C}\left([0, T] ;\left(1+\dot{B}_{p, 1}^{n / p}\right)\right) \times E_{p}(T)$ to $(1.2)$ then one may check (see the appendix of [13]) that, under condition $(2.7)$, the "flow" $X(t, \cdot)$ of $\bar{u}$ defined by

$$
X(t, y):=y+\int_{0}^{t} \bar{v}(\tau, y) d \tau
$$

is a $C^{1}$ diffeomorphism over $\mathbb{R}^{n}$, and satisfies $D X-\operatorname{Id} \in \mathcal{C}\left([0, T] ; \dot{B}_{p, 1}^{n / p}\right)$. Hence one may construct the Eulerian vector-field $u$ and Eulerian density by setting

$$
\rho(t, \cdot):=\rho \circ X^{-1}(t, \cdot) \text { and } u(t, \cdot):=u \circ X^{-1}(t, \cdot) .
$$

As above, the algebraic relations (A.5), (A.6), (A.7) and (A.8) hold whenever $p<2 n$. Hence $(\rho, u)$ is a solution to $(0.1)$. That $(\rho, u)$ has the desired regularity stems from Proposition 8.

Proof of Theorem 2. We consider data $\left(\rho_{0}, u_{0}\right)$ with $\rho_{0}$ bounded away from $0,\left(\rho_{0}-1\right) \in$ $\dot{B}_{p, 1}^{n / p}$ and $u_{0} \in \dot{B}_{p, 1}^{n / p-1}$ Then Theorem 1 provides a local solution $(\bar{\rho}, \bar{u})$ to System (1.2) in $\mathcal{C}\left([0, T] ;\left(1+\dot{B}_{p, 1}^{n / p}\right)\right) \times E_{p}(T)$. If $T$ is small enough then (3.32) is satisfied so Proposition 7 ensures that $\left(\bar{\rho} \circ X^{-1}, \bar{u} \circ X^{-1}\right)$ is a solution of (0.1) in the desired functional space. 
In order to prove uniqueness, we consider two solutions $\left(\rho^{1}, u^{1}\right)$ and $\left(\rho^{2}, u^{2}\right)$ corresponding to the same data $\left(\rho_{0}, u_{0}\right)$, and perform the Lagrangian change of variable (pertaining to the flow of $u^{1}$ and $u^{2}$ respectively). The obtained vector-fields $\bar{u}^{1}$ and $\bar{u}^{2}$ are in $E_{p}(T)$ and both satisfy (1.2) with the same $\rho_{0}$ and $u_{0}$. Hence they coincide, as a consequence of the uniqueness part of Theorem 1.

\section{Appendix A}

A.1. Change of coordinates. Here we establish a result of regularity concerning changes of variables in Besov spaces. Even though this is somewhat classical (at least in nonhomogeneous Besov spaces), we did not find any reference in the literature of the estimates that we need. We here give a result in general Besov spaces $\dot{B}_{p, q}^{s}$, the definition of which may be found in e.g. [1].

Proposition 8. Let $X$ be a globally bi-Lipschitz diffeomorphism of $\mathbb{R}^{n}$ and $(s, p, q)$ with $1 \leq$ $p<\infty$ and $-n / p^{\prime}<s<n / p$ (or just $-n / p^{\prime}<s \leq n / p$ if $q=1$ and just $-n / p^{\prime} \leq s<n / p$ if $q=\infty)$.

Then $a \mapsto a \circ X$ is a self-map over $\dot{B}_{p, q}^{s}$ in the following cases:

(1) $s \in(0,1)$,

(2) $s \in(-1,0]$ and $J_{X^{-1}}$ is in the multiplier space $\mathcal{M}\left(\dot{B}_{p^{\prime}, q^{\prime}}^{-s}\right)$ defined in $(2.9)$,

(3) $s \geq 1$ and $(D X-\mathrm{Id}) \in \dot{B}_{p, 1}^{n / p}$.

Proof: Let us first assume that $s \in(0,1)$ and $q=p$. Then one may use the classical characterization of the norm of $\dot{B}_{p, p}^{s}$ in terms of finite differences (see e.g. [1]) so as to write:

$$
\|u \circ X\|_{\dot{B}_{p, p}^{s}\left(\mathbb{R}^{n}\right)}=\left(\int_{\mathbb{R}^{n}} \int_{\mathbb{R}^{n}} \frac{|u(X(y))-u(X(x))|^{p}}{|y-x|^{n+s p}} d y d x\right)^{\frac{1}{p}} .
$$

Hence performing the change of variable $x^{\prime}=X(x)$ and $y^{\prime}=X(y)$, we get

$$
\|u \circ X\|_{\dot{B}_{p, p}^{s}\left(\mathbb{R}^{n}\right)}=\left(\int_{\mathbb{R}^{n}} \int_{\mathbb{R}^{n}} \frac{\left|u\left(y^{\prime}\right)-u\left(x^{\prime}\right)\right|^{p}}{\left|X^{-1}\left(y^{\prime}\right)-X^{-1}\left(x^{\prime}\right)\right|^{n+s p}} J_{X^{-1}}\left(y^{\prime}\right) J_{X^{-1}}\left(x^{\prime}\right) d y^{\prime} d x^{\prime}\right)^{\frac{1}{p}}
$$

whence

$$
\|u \circ X\|_{\dot{B}_{p, 1}^{s}\left(\mathbb{R}^{n}\right)} \leq\left\|J_{X^{-1}}\right\|_{L_{\infty}\left(\mathbb{R}^{n}\right)}^{\frac{2}{p}}\|D X\|_{L_{\infty}\left(\mathbb{R}^{n}\right)}^{s+\frac{n}{p}}\|u\|_{\dot{B}_{p, 1}^{s}\left(\mathbb{R}^{n}\right)} .
$$

The condition that $s<n / p$ ensures in addition that $u$ belongs to some Lebesgue space $L_{p^{*}}\left(\mathbb{R}^{n}\right)$ with $p^{*}<\infty$ (or in the set of continuous functions going to 0 at infinity if $q=1$ and $s=n / p$ ). Hence $u \circ X \in L_{p^{*}}\left(\mathbb{R}^{n}\right)$ too and one may thus conclude that $u \circ X \in \dot{B}_{p, p}^{s}\left(\mathbb{R}^{n}\right)$. An interpolation argument then yields the desired result for any $s \in(0,1)$ and $q \in[1,+\infty]$.

The result for negative $s$ may be achieved by duality: we have

$$
\|u \circ X\|_{\dot{B}_{p, q}^{s}\left(\mathbb{R}^{n}\right)}=\sup _{\|v\|_{\dot{B}_{p^{\prime}, q^{\prime}}^{-s}\left(\mathbb{R}^{n}\right)} \leq 1} \int_{\mathbb{R}^{n}} v(z) u(X(z)) d z .
$$

Now, setting $x=X(z)$, we have

$$
\begin{aligned}
\int_{\mathbb{R}^{n}} v(z) u(X(z)) d z & =\int_{\mathbb{R}^{n}} u(x) v\left(X^{-1}(x)\right) d x \\
& =\int_{\mathbb{R}^{n}} u(x) v\left(X^{-1}(x)\right) J_{X^{-1}}(x) d x \\
& \leq\|u\|_{\dot{B}_{p, q}^{s}\left(\mathbb{R}^{n}\right)} \| v \circ X^{-1} J_{X^{-1} \|_{\dot{B}_{p^{\prime}, q^{\prime}}^{-s}}\left(\mathbb{R}^{n}\right)^{\prime}}
\end{aligned}
$$

So the definition of the multiplier space and the first part of the lemma allows to conclude. 
Finally, let us examine the cases of larger values of $s$. If $1<s<2$ then one may write

$$
D(u \circ X)=(D u \circ X) \cdot D X .
$$

As $0<s-1<1$, the first part of the proof ensures that $D u \circ X \in \dot{B}_{p, q}^{s-1}$. As moreover $(D X-\mathrm{Id}) \in \dot{B}_{p, 1}^{n / p}$, the standard product laws in Besov spaces give the result.

If $2<s<3$ then we use the algebraic relation,

$$
D^{2}(u \circ X)=\left(D^{2} u \circ X\right)(D X, D X)+D^{2} X \cdot(D u \circ X) .
$$

Hence the result follows from product laws and the previous result applied with $s-1$ or $s-2$.

The higher values of $s$ may be achieved by induction, and the remaining cases ( $s$ an integer) follow by interpolation. The details are left to the reader.

A.2. Some properties of Lagrangian coordinates. Let us first derive a few algebraic relations involving changes of coordinates. We are given a $C^{1}$-diffeomorphism $X$ over $\mathbb{R}^{n}$. For $H: \mathbb{R}^{n} \rightarrow \mathbb{R}^{m}$, we agree that $\bar{H}(y)=H(x)$ with $x=X(y)$. With this convention, the chain rule writes

$$
D_{y} \bar{H}(y)=D_{x} H(X(y)) \cdot D_{y} X(y) \quad \text { with }\left(D_{x} H\right)_{i j}=\partial_{x_{j}} H^{i} \text { and }\left(D_{y} X\right)_{i j}=\partial_{y_{j}} X^{i},
$$

or, denoting $\nabla_{y}={ }^{T} D_{y}$ and $\nabla_{x}={ }^{T} D_{x}$,

$$
\nabla_{y} \bar{H}(y)=\left(\nabla_{y} X(y)\right) \cdot \nabla_{x} H(X(y)) .
$$

Hence we have

$$
D_{x} H(x)=D_{y} \bar{H}(y) \cdot A(y) \quad \text { with } \quad A(y)=\left(D_{y} X(y)\right)^{-1}=D_{x} X^{-1}(x) .
$$

Lemma 1. Let $K$ be a $C^{1}$ scalar function over $\mathbb{R}^{n}$ and $H$, a $C^{1}$ vector-field. Let $X$ be a $C^{1}$ diffeomorphism such that $J:=\operatorname{det}\left(D_{y} X\right)>0$. Then the following relations hold true:

$$
\begin{aligned}
& \overline{\nabla_{x} K}=J^{-1} \operatorname{div}_{y}\left(\operatorname{adj}\left(D_{y} X\right) \bar{K}\right), \\
& \overline{\operatorname{div}_{x} H}=J^{-1} \operatorname{div}_{y}\left(\operatorname{adj}\left(D_{y} X\right) \bar{H}\right),
\end{aligned}
$$

where $\operatorname{adj}\left(D_{y} X\right)$ stands for the adjugate of $D_{y} X$.

Proof: The first item stems from the following series of computations (based on integrations by parts, changes of variable and (A.2)) which hold for any vector-field $\phi$ with coefficients in $\mathcal{C}_{c}^{\infty}\left(\mathbb{R}^{n}\right)$ :

$$
\begin{aligned}
\int \nabla_{x} K(x) \cdot \phi(x) d x & =-\int K(x) \operatorname{div}_{x} \phi(x) d x \\
& =-\int \bar{K}(y) \overline{\operatorname{div}_{x} \phi}(y) J(y) d y \\
& =-\int J(y) \bar{K}(y) D_{y} \bar{\phi}(y): A(y) d y \\
& =\int \bar{\phi}(y) \cdot \operatorname{div}_{y}\left(\operatorname{adj}\left(D_{y} X\right) \bar{K}\right)(y) d y \\
& =\int \phi(x) \cdot \operatorname{div}_{y}\left(\operatorname{adj}\left(D_{y} X\right) \bar{K}\right)\left(X^{-1}(x)\right) J^{-1}\left(X^{-1}(x)\right) d x
\end{aligned}
$$

Proving the second item is similar.

Combining (A.2), (A.4) and (A.3), we deduce that if $u: \mathbb{R}^{n} \rightarrow \mathbb{R}^{n}$ and $P: \mathbb{R}^{n} \rightarrow \mathbb{R}$ then

$$
\begin{aligned}
& \overline{\Delta_{x} u}=J^{-1} \operatorname{div}_{y}\left(\operatorname{adj}\left(D_{y} X\right) \overline{\nabla_{x} u}\right)=J^{-1} \operatorname{div}_{y}\left(\operatorname{adj}\left(D_{y} X\right)^{T} A \nabla_{y} \bar{u}\right), \\
& \overline{\nabla_{x} \operatorname{div}_{x} u}=J^{-1} \operatorname{div}_{y}\left(\operatorname{adj}\left(D_{y} X\right) \overline{\operatorname{div}_{x} u}\right)=J^{-1} \operatorname{div}_{y}\left(\operatorname{adj}\left(D_{y} X\right)^{T} A: \nabla_{y} \bar{u}\right), \\
& \overline{\nabla_{x} P}=J^{-1} \operatorname{div}_{y}\left(\operatorname{adj}\left(D_{y} X\right) \bar{P}\right) .
\end{aligned}
$$


Note that we will use the above relations in the case where $X$ is the flow of some time-dependent vector field $u$, defined by the relation

$$
X(t, y)=y+\int_{0}^{t} u(\tau, X(\tau, y)) d \tau \text { for all } t \in[0, T]
$$

Hence we will also have

$$
J \overline{\partial_{t} \rho+\operatorname{div}(\rho u)}=\partial_{t}(J \bar{\rho}) \quad \text { and } \quad J \overline{\partial_{t}(\rho u)+\operatorname{div}(\rho u \otimes u)}=\partial_{t}(J \bar{\rho} \bar{u})
$$

Let us now establish some estimates for the flow $X_{v}$ of some given "Lagrangian" vector field (that is $X_{v}$ is defined by (3.33)).

Lemma 2. Let $p \in[1,+\infty)$ and $\bar{v}$ be in $E_{p}(T)$ satisfying (2.7). Let $X_{v}$ be defined by (3.33). Then we have for all $t \in[0, T]$,

$$
\begin{aligned}
& \left\|\operatorname{Id}-\operatorname{adj}\left(D X_{v}(t)\right)\right\|_{\dot{B}_{p, 1}^{n / p}} \lesssim\|D \bar{v}\|_{L_{t}^{1}\left(\dot{B}_{p, 1}^{n / p}\right)}, \\
& \left\|\operatorname{Id}-A_{v}(t)\right\|_{\dot{B}_{p, 1}^{n / p}} \lesssim\|D \bar{v}\|_{L_{t}^{1}\left(\dot{B}_{p, 1}^{n / p}\right)}, \\
& \left\|J_{v}^{ \pm 1}(t)-1\right\|_{\dot{B}_{p, 1}^{n / p}} \lesssim\|D \bar{v}\|_{L_{t}^{1}\left(\dot{B}_{p, 1}^{n / p}\right)} .
\end{aligned}
$$

Furthermore, if $\bar{w}$ is a vector field such that $D \bar{w} \in L^{1}\left(0, T ; \dot{B}_{p, 1}^{n / p}\right)$ then

$$
\begin{aligned}
&\left\|\left(\operatorname{adj}\left(D X_{v}\right) D_{A_{v}}(\bar{w})-D(\bar{w})\right)(t)\right\|_{\dot{B}_{p, 1}^{n / p}} \lesssim\|D \bar{v}\|_{L_{t}^{1}\left(\dot{B}_{p, 1}^{n / p}\right)}\|D \bar{w}\|_{L_{t}^{1}\left(\dot{B}_{p, 1}^{n / p}\right)}, \\
&\left\|\left(\operatorname{adj}\left(D X_{v}\right) \operatorname{div}_{A_{v}}(\bar{w})-\operatorname{div} \bar{w} \operatorname{Id}\right)(t)\right\|_{\dot{B}_{p, 1}^{n / p}} \lesssim\|D \bar{v}\|_{L_{t}^{1}\left(\dot{B}_{p, 1}^{n / p}\right)}\|D \bar{w}\|_{L_{t}^{1}\left(\dot{B}_{p, 1}^{n / p}\right)} .
\end{aligned}
$$

Proof: Recall that (see e.g. the appendix of [14]) for any $n \times n$ matrix $C$ we have

$$
\operatorname{Id}-\operatorname{adj}(\operatorname{Id}+C)=(C-(\operatorname{Tr} C) \operatorname{Id})+P_{2}(C),
$$

where the entries of the matrix $P_{2}(C)$ are at least quadratic polynomials. Applying this relation to the matrix $D X(t)$, and using the fact that

$$
D X_{v}(t, y)-\mathrm{Id}=\int_{0}^{t} D \bar{v}(\tau, y) d \tau
$$

we deduce that

$$
\operatorname{Id}-\operatorname{adj}\left(D X_{v}(t)\right)=\int_{0}^{t}(D \bar{v}-\operatorname{div} \bar{v} \operatorname{Id}) d \tau+P_{2}\left(\left(\int_{0}^{t} D \bar{v} d \tau\right)\right) .
$$

Given that $\dot{B}_{p, 1}^{n / p}$ is a Banach algebra and that (2.7) holds, we readily get (A.9).

In order to prove (A.10), we just use the fact that, under assumption (2.7), we have

$$
A_{v}(t)=\left(\operatorname{Id}+C_{v}(t)\right)^{-1}=\sum_{k \in \mathbb{N}}(-1)^{k}\left(C_{v}(t)\right)^{k} \quad \text { with } \quad C_{v}(t)=\int_{0}^{t} D \bar{v} d \tau,
$$

and that $\dot{B}_{p, 1}^{n / p}$ is a Banach algebra.

As regards (A.11), we write

$$
J_{v}(t, y)=1+\int_{0}^{t} \operatorname{div} v\left(\tau, X_{v}(\tau, y)\right) J_{v}(\tau, y) d \tau=1+\int_{0}^{t}\left(D \bar{v}: \operatorname{adj}\left(D X_{v}\right)\right)(\tau, y) d \tau .
$$

Hence, if Condition (2.7) holds then we have (A.11) for $J_{v}$. In order to get the inequality for $J_{v}^{-1}$, it suffices to use the fact that

$$
J_{v}^{-1}(t, y)-1=\left(1+\left(J_{v}(t, y)-1\right)\right)^{-1}-1=\sum_{k \geq 1}(-1)^{k}\left(\int_{0}^{t} D \bar{v}: \operatorname{adj}\left(D X_{v}\right) d \tau\right)^{k}
$$


For proving (A.12), we use the decomposition

$$
\begin{aligned}
2\left(\operatorname{adj}\left(D X_{v}\right) D_{A_{v}}(\bar{w})-D(\bar{w})\right)=\left(\operatorname{adj}\left(D X_{v}\right)-\operatorname{Id}\right)(D \bar{w}+\nabla \bar{w}) & \\
& +\left(\operatorname{adj}\left(D X_{v}\right)-\operatorname{Id}\right)\left(D \bar{w} \cdot(A-\operatorname{Id})+\left({ }^{T} A-\operatorname{Id}\right) \cdot \nabla \bar{w}\right) .
\end{aligned}
$$

Hence the desired inequality stems from (A.9) and (A.10), and from the fact that $\dot{B}_{p, 1}^{n / p}$ is a Banach algebra. Inequality (A.13) is similar. This completes the proof of the lemma.

Lemma 3. Let $\bar{v}_{1}$ and $\bar{v}_{2}$ be two vector-fields satisfying (2.7), and $\delta v:=\bar{v}_{2}-\bar{v}_{1}$. Then we have for all $p \in[1,+\infty)$ and all $t \in[0, T]$ (with obvious notation):

$$
\begin{gathered}
\left\|A_{2}(t)-A_{1}(t)\right\|_{\dot{B}_{p, 1}^{n / p}} \lesssim\|D \delta v\|_{L_{t}^{1}\left(\dot{B}_{p, 1}^{n / p}\right)}, \\
\left\|\operatorname{adj}\left(D X_{2}(t)\right)-\operatorname{adj}\left(D X_{1}(t)\right)\right\|_{\dot{B}_{p, 1}^{n / p}} \lesssim\|D \delta v\|_{L_{t}^{1}\left(\dot{B}_{p, 1}^{n / p}\right)}, \\
\left\|J_{2}^{ \pm 1}(t)-J_{1}^{ \pm 1}(t)\right\|_{\dot{B}_{p, 1}^{n / p}} \lesssim\|D \delta v\|_{L_{t}^{1}\left(\dot{B}_{p, 1}^{n / p}\right)} .
\end{gathered}
$$

Proof: In order to prove the first inequality, we use the fact that, for $i=1,2$, we have

$$
A_{i}=\left(\operatorname{Id}+C_{i}\right)^{-1}=\sum_{k \geq 0}(-1)^{k} C_{i}^{k} \quad \text { with } \quad C_{i}(t)=\int_{0}^{t} D \bar{v}_{i} d \tau .
$$

Hence

$$
A_{2}-A_{1}=\sum_{k \geq 1}\left(C_{2}^{k}-C_{1}^{k}\right)=\left(\int_{0}^{t} D \delta v d \tau\right) \sum_{k \geq 1} \sum_{j=0}^{k-1} C_{1}^{j} C_{2}^{k-1-j} .
$$

So using the fact that $\dot{B}_{p, 1}^{n / p}$ is a Banach algebra, it is easy to conclude to (A.18).

The second inequality is a consequence of the decomposition (A.14) and of the Taylor formula which ensures that, denoting $\delta C:=C_{2}-C_{1}$,

$$
\operatorname{adj}\left(D X_{2}\right)-\operatorname{adj}\left(D X_{1}\right)=(\operatorname{Tr}(\delta C)) \operatorname{Id}-\delta C+d P_{2}\left(C_{1}\right)(\delta C)+\frac{1}{2} d^{2} P_{2}\left(C_{1}, C_{1}\right)(\delta C, \delta C)+\cdots
$$

where the coefficients of $P_{2}$ are polynomials of degree $n-1$. As the sum is finite and $\dot{B}_{p, 1}^{n / p}$ is a Banach algebra, we get (A.19).

Proving the third inequality relies on similar arguments. It is only a matter of using (A.17). The details are left to the reader.

A.3. Commutator and product estimates. This last paragraph is devoted to the proof of commutator and product estimates that have been used for investigating the Lamé system. Those proofs rely on the following Bony decomposition (first introduced in [2]) for the product of two functions:

$$
f g=T_{f} g+R(f, g)+T_{g} f .
$$

The paraproduct and remainder operators $T$ and $R$ are defined by

$$
T_{f} g:=\sum_{j^{\prime} \leq j-2} \dot{\Delta}_{j^{\prime}} f \dot{\Delta}_{j} g \text { and } R(f, g):=\sum_{\left|j^{\prime}-j\right| \leq 1} \dot{\Delta}_{j^{\prime}} f \dot{\Delta}_{j} g
$$

where $\left(\dot{\Delta}_{j}\right)_{j \in \mathbb{Z}}$ stands for some homogeneous Littlewood-Paley decomposition. 
Lemma 4. Let $p$ be in $[1,+\infty]$ and the real numbers $\nu$ and $\sigma$ satisfy

$$
\nu \geq 0 \quad \text { and } \quad-\min \left(\frac{n}{p}, \frac{n}{p^{\prime}}\right)<\sigma \leq \frac{n}{p}-\nu .
$$

Then the following estimate holds true for all tempered distributions $f$ and $g$ over $\mathbb{R}^{n}$ :

$$
\|f g\|_{\dot{B}_{p, 1}^{\sigma}} \lesssim\|f\|_{\dot{B}_{p, 1}^{n / p-\nu}}\|g\|_{\dot{B}_{p, 1}^{\sigma+\nu}}
$$

Proof. The result relies on Bony decomposition (A.21). The standard continuity results for the paraproduct and remainder operators ensure that (see e.g. [1], Chap. 2):

$$
\begin{aligned}
\left\|T_{f} g\right\|_{\dot{B}_{p, 1}^{\sigma}} & \lesssim\|f\|_{\dot{B}_{\infty, 1}^{-\nu}}\|g\|_{\dot{B}_{p, 1}^{\sigma+\nu}} & & \text { if } \nu \geq 0, \\
\left\|T_{g} f\right\|_{\dot{B}_{p, 1}^{\sigma}} & \lesssim\|g\|_{\dot{B}_{\infty, 1}^{\sigma+\nu-n / p}}\|f\|_{\dot{B}_{p, 1}^{n / p-\nu}} & & \text { if } \sigma+\nu-n / p \leq 0, \\
\|R(f, g)\|_{\dot{B}_{p, 1}^{\sigma}} & \lesssim\|f\|_{\dot{B}_{p, 1}^{n / p-\nu}}\|g\|_{\dot{B}_{p, 1}^{\sigma+\nu}} & & \text { if } \sigma>-\min \left(n / p, n / p^{\prime}\right) .
\end{aligned}
$$

So the result follows once noticed that $\dot{B}_{p, 1}^{s} \hookrightarrow \dot{B}_{\infty, 1}^{s-n / p}$ for any $s \in \mathbb{R}$.

Lemma 5. Assume that $\sigma, \nu$ and $p$ are such that

$$
1 \leq p \leq+\infty, \quad 0 \leq \nu \leq \frac{n}{p} \text { and }-\min \left(\frac{n}{p}, \frac{n}{p^{\prime}}\right)-1<\sigma \leq \frac{n}{p}-\nu .
$$

There exists a constant $C$ depending only on $\nu, p, \sigma$ and $n$ such that for all $k \in\{1, \cdots, n\}$, we have for some sequence $\left(c_{j}\right)_{j \in \mathbb{Z}}$ with $\|c\|_{\ell^{1}(\mathbb{Z})}=1$ :

$$
\left\|\partial_{k}\left[a, \dot{\Delta}_{j}\right] w\right\|_{L^{p}} \leq C c_{j} 2^{-j \sigma}\|\nabla a\|_{B_{p, 1}^{n / p-\nu}}\|w\|_{B_{p, 1}^{\sigma+\nu}} \quad \text { for all } j \in \mathbb{Z} .
$$

Proof. Taking advantage of the Bony decomposition (A.21), we rewrite the commutator as $^{3}$

$$
\partial_{k}\left(\left[a, \dot{\Delta}_{j}\right] w\right)=\underbrace{\partial_{k}\left(\left[T_{a}, \dot{\Delta}_{j}\right] w\right)}_{R_{j}^{1}}+\underbrace{\partial_{k} T_{\dot{\Delta}_{j} w}^{\prime} a}_{R_{j}^{2}}-\underbrace{\partial_{k} \dot{\Delta}_{j} T_{w}^{\prime} a}_{R_{j}^{3}} .
$$

Arguing as in the proof of Lemma 6 in [12], we get

$$
\left\|R_{j}^{1}\right\|_{L^{p}} \leq C \sum_{\left|j^{\prime}-j\right| \leq 4}\left\|\nabla \dot{S}_{j^{\prime}-1} a\right\|_{L^{\infty}}\left\|\dot{\Delta}_{j^{\prime}} w\right\|_{L^{p}}
$$

Now, for $\nu \geq 0$, we have

$$
\left\|\nabla \dot{S}_{j^{\prime}-1} a\right\|_{L^{\infty}} \leq C 2^{j^{\prime} \nu}\|\nabla a\|_{\dot{B}_{\infty, 1}^{-\nu}}
$$

Therefore, for some sequence $\left(c_{j}\right)_{j \in \mathbb{Z}}$ in the unit sphere of $\ell^{1}(\mathbb{Z})$,

$$
\left\|R_{j}^{1}\right\|_{L^{p}} \leq C c_{j} 2^{-j \sigma}\|\nabla a\|_{\dot{B}_{\infty, 1}^{-\nu}}\|w\|_{\dot{B}_{p, 1}^{\sigma+\nu}} .
$$

To deal with $R_{j}^{2}$, we use the fact that, owing to the localization properties of the LittlewoodPaley decomposition, we have

$$
R_{j}^{2}=\sum_{j^{\prime} \geq j-2} \partial_{k}\left(S_{j^{\prime}+2} \dot{\Delta}_{j} w \dot{\Delta}_{j^{\prime}} a\right)
$$

Hence, using the Bernstein and Hölder inequalities,

$$
\begin{aligned}
\left\|R_{j}^{2}\right\|_{L^{p}} & \leq C \sum_{j^{\prime} \geq j-2}\left\|S_{j^{\prime}+2} \dot{\Delta}_{j} w\right\|_{L^{\infty}}\left\|\dot{\Delta}_{j^{\prime}} \nabla a\right\|_{L^{p}}, \\
& \leq C 2^{-j \sigma} \sum_{j^{\prime} \geq j-2} 2^{\left(j-j^{\prime}\right)\left(\frac{n}{p}-\nu\right)}\left(2^{j\left(\sigma+\nu-\frac{n}{p}\right)}\left\|\dot{\Delta}_{j} w\right\|_{L^{\infty}}\right)\left(2^{j^{\prime}\left(\frac{n}{p}-\nu\right)}\left\|\dot{\Delta}_{j^{\prime}} \nabla a\right\|_{L^{p}}\right) .
\end{aligned}
$$

\footnotetext{
${ }^{3}$ Here we use the notation $T_{u}^{\prime} v:=T_{u} v+R(u, v)$.
} 
Therefore, by virtue of convolution inequalities for series and because $n / p-\nu \geq 0$,

$$
\left\|R_{j}^{2}\right\|_{L^{p}} \leq C c_{j} 2^{-j \sigma}\|\nabla a\|_{B_{p, 1}^{n / p-\nu}}\|w\|_{B_{\infty, 1}^{\sigma+\nu-\frac{n}{p}}} .
$$

Next, from standard continuity results, we know that the paraproduct and the remainder map $B_{p, 1}^{\sigma+\nu} \times B_{p, 1}^{n / p-\nu+1}$ in $B_{p, 1}^{\sigma+1}$ whenever $\sigma+\nu-n / p \leq 0$ and $\sigma+1>-\min \left(n / p, n / p^{\prime}\right)$. We thus have

$$
\left\|R_{j}^{3}\right\|_{L^{p}} \leq C c_{j} 2^{-j \sigma}\|\nabla a\|_{B_{p, 1}^{n / p-\nu}}\|w\|_{B_{p, 1}^{\sigma+\nu}}
$$

Putting Inequalities (A.24), (A.25) and (A.26) together, and using classical embedding completes the proof of the lemma.

Lemma 6. Let $A(D)$ be a Fourier multiplier of degree 0. Then the following estimate holds

$$
\|[A(D), q] w\|_{\dot{B}_{p, 1}^{\sigma+1}} \leq C\|q\|_{\dot{B}_{p, 1}^{1-\nu+n / p}}\|w\|_{\dot{B}_{p, 1}^{\sigma+\nu}}
$$

whenever

$$
\nu \geq 0 \quad \text { and } \quad-\min \left(\frac{n}{p}, \frac{n}{p^{\prime}}\right)-1<\sigma \leq \frac{n}{p}-\nu .
$$

Proof. Taking advantage once again of Bony's decomposition, we decompose the commutator into

$$
[A(D), q] w=\left[A(D), T_{q}\right] w+A(D) T_{w}^{\prime} q-T_{A(D) w}^{\prime} q .
$$

According to Lemma 2.99 in $[1]$, we have for $\nu \geq 0$,

$$
\left\|\left[A(D), T_{q}\right] w\right\|_{\dot{B}_{p, 1}^{\sigma+1}} \leq C\|\nabla q\|_{\dot{B}_{\infty, 1}^{-\nu}}\|w\|_{\dot{B}_{p, 1}^{\sigma+\nu}}
$$

Next, given that $A(D)$ is a homogeneous multiplier of degree 0, it maps any homogeneous Besov space in itself. Therefore the last two terms of (A.27) may be just bounded according to standard continuity results for the paraproduct and remainder operators.

\section{REFERENCES}

[1] H. Bahouri, J.-Y. Chemin and R. Danchin: Fourier Analysis and Nonlinear Partial Differential Equations, Grundlehren der mathematischen Wissenschaften, 343, Springer (2011).

[2] J.-M. Bony: Calcul symbolique et propagation des singularités pour les équations aux dérivées partielles non linéaires, Annales Scientifiques de l'École Normale Supérieure, 14, 209-246 (1981).

[3] F. Charve and R. Danchin: A global existence result for the compressible Navier-Stokes equations in the critical $L^{p}$ framework, Archive for Rational Mechanics and Analysis, 198(1), 233-271 (2010).

[4] Q. Chen, C. Miao and Z. Zhang: Well-posedness in critical spaces for the compressible Navier-Stokes equations with density dependent viscosities, Revista Matemática Iberoamericana, 26(3), 915-946 (2010).

[5] Q. Chen, C. Miao and Z. Zhang: Global well-posedness for the compressible Navier-Stokes equations with the highly oscillating initial velocity, Communications on Pure and Applied Mathematics, 63(9), 1173-1224 (2010).

[6] Y. Cho, H.J. Choe and H. Kim: Unique solvability of the initial boundary value problems for compressible viscous fluids, Journal de Mathématiques Pures et Appliquées, 83(2), 243-275 (2004).

[7] R. Danchin: Global existence in critical spaces for compressible Navier-Stokes equations, Inventiones Mathematicae, 141(3), 579-614 (2000).

[8] R. Danchin: Local theory in critical spaces for compressible viscous and heat-conductive gases, Communications in Partial Differential Equations, 26, 1183-1233 (2001).

[9] R. Danchin: On the uniqueness in critical spaces for compressible Navier-Stokes equations, NoDEA Nonlinear Differential Equations Appl., 12(1), 111-128 (2005).

[10] R. Danchin: Well-posedness in critical spaces for barotropic viscous fluids with truly nonconstant density, Communications in Partial Differential Equations, 32, 1373-1397 (2007).

[11] R. Danchin: On the solvability of the compressible Navier-Stokes system in bounded domains, Nonlinearity, 23, 383-407 (2010).

[12] R. Danchin: On the well-posedness of the incompressible density-dependent Euler equations in the $L^{p}$ framework, Journal of Differential Equations, 248, 2130-2170 (2010). 
[13] R. Danchin: Fourier analysis methods for compressible flows, topics on compressible Navier-Stokes equations, états de la recherche SMF, Chambéry, 2012.

[14] R. Danchin and P. B. Mucha: A Lagrangian approach for the incompressible Navier-Stokes equations with variable density, Communications on Pure and Applied Mathematics, 65, 1458-1480 (2012).

[15] P. Germain: Weak-strong uniqueness for the isentropic compressible Navier-Stokes system, J. Math. Fluid Mech., 13(1), 137-146 (2011).

[16] B. Haspot: Existence of global strong solutions in critical spaces for barotropic viscous fluids, Archive for Rational Mechanics and Analysis, 202, 427-460 (2011).

[17] B. Haspot: Well-posedness in critical spaces for the system of compressible Navier-Stokes in larger spaces, Journal of Differential Equations, 251, 2262-2295 (2011).

[18] D. Hoff: Uniqueness of weak solutions of the Navier-Stokes equations of multidimensional compressible flow, SIAM Journal on Mathematical Analysis, 37(6), 1742-1760 (2006).

[19] N. Krylov: Lectures on elliptic and parabolic equations in Sobolev spaces, Graduate studies in Mathematics, 96, American Mathematical Society, 2008.

[20] P.-L. Lions: Mathematical Topics in Fluid Dynamics, Vol. 2 Compressible Models, Oxford University Press (1998).

[21] P. Mucha: The Cauchy problem for the compressible Navier-Stokes equations in the $L^{p}$-framework, Nonlinear Analysis, 52(4), 1379-1392 (2003).

[22] A. Valli: An existence theorem for compressible viscous fluids, Annali di Matematica Pura ed Applicata, 130 (4), 197-213 (1982).

[23] A. Valli and W. Zajączkowski: Navier-Stokes equations for compressible fluids: global existence and qualitative properties of the solutions in the general case, Communications in Mathematical Physics, 103(2), 259-296 (1986).

(R. Danchin) Université Paris-Est, LAMA, UMR 8050 and Institut Universitaire de France, 61 avenue du Général de Gaulle, 94010 Créteil Cedex, France.

E-mail address: danchin@univ-paris12.fr 